\title{
Effects of Rotation and Gravity Field on Surface Waves in Fibre-Reinforced Thermoelastic Media under Four Theories
}

\author{
A. M. Abd-Alla, ${ }^{1,2}$ S. M. Abo-Dahab, ${ }^{1,3}$ and A. Al-Mullise ${ }^{1}$ \\ ${ }^{1}$ Mathematics Department, Faculty of Science, Taif University 888, Saudi Arabia \\ ${ }^{2}$ Mathematics Department, Faculty of Science, Sohag University, Egypt \\ ${ }^{3}$ Mathematics Department, Faculty of Science, Qena 83523, Egypt \\ Correspondence should be addressed to A. M. Abd-Alla; mohmrr@yahoo.com
}

Received 4 August 2013; Accepted 22 October 2013

Academic Editor: Morteza Rafei

Copyright (C) 2013 A. M. Abd-Alla et al. This is an open access article distributed under the Creative Commons Attribution License, which permits unrestricted use, distribution, and reproduction in any medium, provided the original work is properly cited.

\begin{abstract}
Estimation is done to investigate the gravitational and rotational parameters effects on surface waves in fibre-reinforced thermoelastic media. The theory of generalized surface waves has been firstly developed and then it has been employed to investigate particular cases of waves, namely, Stoneley waves, Rayleigh waves, and Love waves. The analytical expressions for surface waves velocity and attenuation coefficient are obtained in the physical domain by using the harmonic vibrations and four thermoelastic theories. The wave velocity equations have been obtained in different cases. The numerical results are given for equation of coupled thermoelastic theory (C-T), Lord-Shulman theory (L-S), Green-Lindsay theory (G-L), and the linearized (G-N) theory of type II. Comparison was made with the results obtained in the presence and absence of gravity, rotation, and parameters for fibre-reinforced of the material media. The results obtained are displayed by graphs to clear the phenomena physical meaning. The results indicate that the effect of gravity, rotation, relaxation times, and parameters of fibre-reinforced of the material medium is very pronounced.
\end{abstract}

\section{Introduction}

A reinforced concrete member will be designed for all conditions of stress that may occur accordance with the principle of mechanics. Fibre-reinforced composites are used in a variety of structures due to their low weight and high strength. The characteristic property of a reinforced composite is that its components act together as single anisotropic units as long as they remain in the elastic condition. Investigation of interaction between magnetic field, stress, and strain in a thermoelastic solid is very important due to its many applications in the field of geophysics, plasma physics, and related topics, especially in the nuclear field, where the extremely high temperature and temperature gradients, as well as the magnetic fields originating inside nuclear reactors, influence their design. Recently, more attention has been studied the dynamical problem of propagation of surface waves in a homogeneous and non-homogeneous elastic and thermoplastic media because of its utilitarian aspects in earthquake, engineering, and seismology on account of the occurrence of nonhomogeneities in the Earth's crust, as the Earth is made up of different layers. Abd-Alla et al. [1] investigated propagation of Rayleigh waves in generalized magnetothermoelastic orthotropic material under initial stress and gravity field. Thermal stresses in an infinite circular cylinder have been investigated by Abd-Alla et al. [2]. Abd-Alla and Mahmoud [3] studied the magnetothermoelastic problem in rotational nonhomogeneous orthotropic hollow cylinder under the hyperbolic heat conduction model. Abd-Alla and Ahmed [4] investigated the Rayleigh waves in an orthotropic thermoelastic medium under gravity field and initial stress. Rayleigh waves in a magnetoelastic half-space of orthotropic material under the influence of initial stress and gravity field have been investigated by Abd-Alla et al. [5]. Elnaggar and Abd-Alla [6] studied Rayleigh waves in magneto-thermomicroelastic halfspace under initial stress. Abd-Alla and Ahmed [7] discussed Rayleigh waves in an orthotropic thermoelastic medium under gravity field and initial stress. Effect of rotation and initial stress on an infinite generalized magnetothermoelastic diffusion body with a spherical cavity has been investigated by Abd-Alla and Abo-Dahab [8]. Wu and Chai [9] studied propagation of surface waves in anisotropic solids: theoretical 
calculation and experiment. Wu and Liu [10] investigated the measurement of anisotropic elastic constants of fibrereinforced composite plate by using ultrasonic bulk wave and laser generated Lamb wave. The group velocity variation of Lamb wave in Fibre-reinforced composite plate studied by Rhee et al. [11]. Fu and Zhang [12] investigated the continuum-mechanical modeling of kink-band formation in fibre-reinforced composites. Espinosa et al. [13] discussed the modeling impact induced delamination of woven Fibrereinforced composites with contact/cohesive laws. Wave propagation in materials reinforced with bi-directional Fibres presented by Weitsman and Benveniste [14]. Weitsman [15] introduced the wave propagation and energy scattering in materials reinforced by inextensible Fibres. Dai and Wang [16] considered the stress wave propagation in piezoelectric Fibre-reinforced laminated composites subjected to thermal shock. Ohyoshi [17] studied the propagation of Rayleigh waves along an obliquely cut surface in a directional Fibrereinforced composite. Rogerson [18] investigated the penetration of impact waves in a six-ply fibre composite laminate. Weitsrian [19] studied the reflection of harmonic waves in fibre-reinforced materials. Huang et al. [20] investigated the effect of fibre-matrix interphase on wave propagation along, and scattering from, multilayered fibres in composites. Fu and Zhang [21] discussed the continuum-mechanical modeling of kink-band formation in fibre-reinforced composites. B. Singh and S. J. Singh [22] investigated the reflection of plane waves at the free surface of a fibre-reinforced elastic halfspace. Sengupta and Nath [23] studied the surface waves in fibre-reinforced anisotropic elastic media. Samal and Chattaraj [24] studied the surface wave propagation in Fibrereinforced anisotropic elastic layer between liquid saturated porous half-space and uniform liquid layer. Chatopadhyay et al. [25] investigated the reflection of quasi-P and quasi-SV waves at the free and rigid boundaries of a fibre-reinforced medium. Singh [26] discussed the wave propagation in an incompressible transversely isotropic fibre-reinforced elastic media. Singh [27] studied the wave propagation in thermally conducting linear fibre-reinforced composite materials. AbdAlla et al. [28] studied the thermal stresses in a nonhomogeneous orthotropic elastic multilayered cylinder.

Recently, Othman and Lotfy [29] investigated the effect of magnetic field and rotation of the 2D problem of a fibrereinforced thermoelastic under three theories with influence of gravity. Abouelregal and Abo-Dahab [30] discuss the dual phase lag model on magnetothermoelasticity infinite nonhomogeneous solid having a spherical cavity. Abd-Alla and Abo-Dahab [31] investigated the effect of magnetic field on poroelastic bone model for internal remodeling. AbdAlla et al. $[32,33]$ studied the Rayleigh waves in generalized magneto thermoviscoelastic granular medium under the influence of rotation, gravity field, and initial stress and magnetothermoelastic half-space of a homogeneous orthotropic material under the effect of the rotation, initial stress and gravity field. Abo-Dahab [34] investigated propagation of Stoneley waves in magnetothermoelastic materials with voids and two relaxation times.

The present investigation is devoted to study the propagation of surface waves in fibre-reinforced anisotropic thermoelastic medium subjected to gravity field and relaxation times leading to particular cases such as Rayleigh waves, Love waves and Stoneley waves. The surface wave velocity and attenuation coefficient are obtained in the physical domain by using the harmonic vibrations. The effects of the gravity, relaxation times, and parameters for fibre-reinforced of the material medium on surface waves are studied simultaneously. The analytical expressions for surface waves velocity and attenuation coefficient are represented graphically. Numerical results for the surface waves velocity and attenuation coefficient are given and illustrated graphically in the presence and absence of the gravity field and rotation.

\section{Formulation of the Problem}

We consider homogeneous thermally conducting transversely two fibre-reinforced media. Let $M_{1}$ and $M_{2}$ be two fibre-reinforced elastic thermoelastic semi-infinite solid media. They are perfectly welded in contact to prevent any relative motion or sliding before and after the disturbances and that the continuity of displacement, stress, and so for the hold good across the common boundary surface. Further the mechanical properties of $M_{1}$ are different from those of $M_{2}$. These media extend to an infinite great distance from the origin and are separated by a plane horizontal boundary and $M_{2}$ is to be taken above $M_{1}$. Let $O x y z$ be a set of orthogonal Cartesian coordinates, let $O$ be any point of the plane boundary, and let $O y$ points vertically downward to the medium $M_{1}$. We consider the possibility of a type of wave travelling in the direction $O x$ in such a manner that the disturbance is largely confined to the neighborhood of the boundary and, at any instant, all particles in any line parallel to $y$-axis have equal displacements. These two assumptions conclude that the wave is a surface wave and all partial derivatives with respect to $z$ are zero. Further let us assume that $u, v$ are the components of displacements at any point $(x, y, z)$ at any time $t$. It is also assumed that gravitational field produces a hydrostatic initial stress that is produced by a slow process of creep where the shearing stresses tend to become smaller or vanish after a long period of time.

The equilibrium equation of the initial stress is in the form

$$
\frac{\partial \tau}{\partial x}=0, \quad \frac{\partial \tau}{\partial y}+\rho g=0
$$

The equations and constitutive relations for such medium in the absence of body forces and heat sources are

$$
\begin{aligned}
(\lambda+ & \left.2 \alpha+4 \mu_{L}-2 \mu_{T}+\beta\right) \frac{\partial^{2} u}{\partial x^{2}}+\left(\lambda+\alpha+\mu_{L}\right) \frac{\partial^{2} v}{\partial x \partial y} \\
& +\mu_{L} \frac{\partial^{2} u}{\partial y^{2}}-\beta_{1}\left(1+\vartheta_{0} \frac{\partial}{\partial t}\right) \frac{\partial T}{\partial x}-\rho g \frac{\partial v}{\partial x} \\
= & \rho\left(\frac{\partial^{2} u}{\partial t^{2}}-2 \Omega \frac{\partial v}{\partial t}-\Omega^{2} u\right)
\end{aligned}
$$




$$
\begin{aligned}
&(\lambda+\left.2 \mu_{T}\right) \frac{\partial^{2} v}{\partial y^{2}}+\left(\lambda+\alpha+\mu_{L}\right) \frac{\partial^{2} u}{\partial x \partial y}+\mu_{L} \frac{\partial^{2} v}{\partial x^{2}} \\
&-\beta_{2}\left(1+\vartheta_{0} \frac{\partial}{\partial t}\right) \frac{\partial T}{\partial y}+\rho g \frac{\partial u}{\partial x} \\
&= \rho\left(\frac{\partial^{2} v}{\partial t^{2}}+2 \Omega \frac{\partial u}{\partial t}-\Omega^{2} v\right), \\
& k_{1}^{*}\left(n^{*}+t_{1} \frac{\partial}{\partial t}\right) \frac{\partial^{2} T}{\partial x^{2}}+k_{2}^{*}\left(n^{*}+t_{1} \frac{\partial}{\partial t}\right) \frac{\partial^{2} T}{\partial y^{2}} \\
&=\rho C^{*}\left(n_{1} \frac{\partial}{\partial t}+\tau_{0} \frac{\partial^{2}}{\partial t^{2}}\right) T+T_{0}\left(n_{1} \frac{\partial}{\partial t}+\tau_{0} n_{0} \frac{\partial^{2}}{\partial t^{2}}\right) \\
& \quad \times\left(\beta_{1} \frac{\partial u}{\partial x}+\beta_{2} \frac{\partial v}{\partial y}\right) .
\end{aligned}
$$

\section{Special Cases of Thermoelasticity Theory}

We get that the forms show the behavior under gravity field; the theories are as follows.

(1) The equation of the coupled thermoelasticity (C-T theory) is obtained when

$n^{*}=n_{1}=1, \quad t_{1}=\tau_{0}=\theta_{0}=0, \quad \tau_{0}=0$.

(2) Consider Lord-Shulman theory.

For the Lord-Shulman (L-S) theory

$$
n^{*}=n_{1}=n_{0}=1, \quad t_{1}=\theta_{0}=0, \quad \tau_{0}>0 .
$$

(3) Consider the Green-Lindsay theory. For the Green-Lindsay (G-L) theory

$$
n^{*}=n_{1}=1, \quad n_{0}=t_{1}=0, \quad \theta_{0} \geq \tau_{0}>0 .
$$

(4) Consider the Green-Nagdhi theory.

For the Green- Nagdhi theory (G-N) of type 2,

$$
\begin{gathered}
n^{*}>0, \quad n_{1}=0, \quad n_{0}=1, \\
t_{1}=\vartheta_{0}=0, \quad \tau_{0}=1 .
\end{gathered}
$$

For plane strain deformation in the $x-y$ plane, the components of stress take the form

$$
\begin{gathered}
\tau_{11}=\left(\lambda+2 \alpha+4 \mu_{L}-2 \mu_{T}+\beta\right) \frac{\partial u}{\partial x} \\
+(\lambda+\alpha) \frac{\partial v}{\partial y}-\beta_{1}\left(1+\vartheta_{0} \frac{\partial}{\partial t}\right) T \\
\tau_{12}=\mu_{L} \frac{\partial v}{\partial x}+\mu_{L} \frac{\partial u}{\partial y} \\
\tau_{21}=\mu_{L} \frac{\partial v}{\partial x}+\mu_{L} \frac{\partial u}{\partial y} \\
\tau_{22}=(\lambda+\alpha) \frac{\partial u}{\partial x}+\left(\lambda+2 \mu_{T}\right) \frac{\partial v}{\partial y}-\beta_{2}\left(1+\vartheta_{0} \frac{\partial}{\partial t}\right) T
\end{gathered}
$$

where

$$
\begin{gathered}
\beta_{1}=\left(2 \lambda+3 \alpha+4 \mu_{L}-2 \mu_{T}+\beta\right) \alpha_{1}+(\lambda+\alpha) \alpha_{2}, \\
\beta_{1}=(2 \lambda+\alpha) \alpha_{1}+\left(\lambda+2 \mu_{T}\right) \alpha_{2} .
\end{gathered}
$$

By Helmholtz's theorem [35], the displacement vector $u$ can be written in the form

$$
\vec{u}=\operatorname{grad} \varphi+\operatorname{curl} \vec{\psi},
$$

where the scalar $\varphi$ and the vector $\vec{\psi}$ represent irrotational and rotational parts of the displacement $\vec{u}$. It is possible to take only one component of the vector $\vec{\psi}$ to be nonzero, as

$$
\vec{\psi}=(0,0, \psi) .
$$

From (14a) and (14b) we obtain

$$
u=\frac{\partial \varphi}{\partial x}+\frac{\partial \Psi}{\partial y}, \quad v=\frac{\partial \varphi}{\partial y}-\frac{\partial \Psi}{\partial x} .
$$

Substituting from (14c) into (2) and (3) we get

$$
\begin{aligned}
& \frac{\left(\lambda+2 \alpha+4 \mu_{L}-2 \mu_{T}+\beta\right)}{\rho} \frac{\partial^{2} \varphi}{\partial x^{2}}+\frac{\left(\lambda+\alpha+2 \mu_{L}\right)}{\rho} \frac{\partial^{2} \varphi}{\partial y^{2}} \\
& -\beta_{1}\left(1+\vartheta_{0} \frac{\partial}{\partial t}\right) T+g \frac{\partial \Psi}{\partial x} \\
& =\frac{\partial^{2} \varphi}{\partial t^{2}}+2 \Omega \frac{\partial \Psi}{\partial t}-\Omega^{2} \varphi, \\
& \frac{\left(\alpha+3 \mu_{L}-2 \mu_{T}+\beta\right)}{\rho} \frac{\partial^{2} \Psi}{\partial x^{2}}+\frac{\mu_{L}}{\rho} \frac{\partial^{2} \Psi}{\partial y^{2}}-g \frac{\partial \varphi}{\partial x} \\
& =\frac{\partial^{2} \Psi}{\partial t^{2}}-2 \Omega \frac{\partial \varphi}{\partial t}-\Omega^{2} \Psi, \\
& \frac{\left(\lambda+\alpha+2 \mu_{L}\right)}{\rho}\left(\frac{\partial^{2} \varphi}{\partial x^{2}}\right)+\frac{\left(\lambda+2 \mu_{T}\right)}{\rho}\left(\frac{\partial^{2} \varphi}{\partial y^{2}}\right) \\
& -\frac{\beta_{2}}{\rho}\left(1+\vartheta_{0} \frac{\partial}{\partial t}\right) T+g \frac{\partial \Psi}{\partial x}=\frac{\partial^{2} \varphi}{\partial t^{2}}+2 \Omega \frac{\partial \Psi}{\partial t}-\Omega^{2} \varphi, \\
& -\frac{\mu_{L}}{\rho}\left(\frac{\partial^{2} \Psi}{\partial x^{2}}\right)+\frac{\left(\alpha+\mu_{L}-2 \mu_{T}\right)}{\rho}\left(\frac{\partial^{2} \Psi}{\partial y^{2}}\right)+g \frac{\partial \varphi}{\partial x} \\
& =-\frac{\partial^{2} \Psi}{\partial t^{2}}+2 \Omega \frac{\partial \varphi}{\partial t}+\Omega^{2} \Psi .
\end{aligned}
$$

From (16) and (18) we get

$$
\frac{\partial^{2} \Psi}{\partial x^{2}}+f_{1} \frac{\partial^{2} \Psi}{\partial y^{2}}=0
$$

where

$$
f_{1}=\frac{\left(\alpha+2 \mu_{L}-2 \mu_{T}\right)}{\left(\alpha+2 \mu_{L}-2 \mu_{T}+\beta\right)} \text {. }
$$


Also from (15) and (17) we get

$$
\begin{gathered}
\frac{\partial^{2} \varphi}{\partial x^{2}}+f_{2} \frac{\partial^{2} \varphi}{\partial y^{2}}-f_{3}\left(1+\vartheta_{0} \frac{\partial}{\partial t}\right) T+f_{4} \frac{\partial \Psi}{\partial x} \\
=f_{5}\left(\frac{\partial^{2} \varphi}{\partial t^{2}}+2 \Omega \frac{\partial \Psi}{\partial t}-\Omega^{2} \varphi\right),
\end{gathered}
$$

where

$$
\begin{aligned}
& f_{2}=\frac{\left(2 \lambda+\alpha+2 \mu_{L}-2 \mu_{T}\right)}{\left(2 \lambda+3 \alpha+6 \mu_{L}-2 \mu_{T}+\beta\right)}, \\
& f_{3}=\frac{\left(\beta_{2}+\beta_{1}\right)}{\left(2 \lambda+3 \alpha+6 \mu_{L}-2 \mu_{T}+\beta\right)}, \\
& f_{4}=\frac{2 g}{2 \lambda+3 \alpha+6 \mu_{L}-2 \mu_{T}+\beta}, \\
& f_{5}=\frac{2}{\left(2 \lambda+3 \alpha+6 \mu_{L}-2 \mu_{T}+\beta\right)} .
\end{aligned}
$$

The solution of (19) and (21) has the form

$$
\begin{aligned}
& \varphi=F(y) e^{i \omega(x-c t)}, \\
& \Psi=G(y) e^{i \omega(x-c t)}, \\
& T=H(y) e^{i \omega(x-c t)} .
\end{aligned}
$$

Substituting from (23) in to (19) we get

$$
G^{\prime \prime}-m^{2} G=0 .
$$

The solution for (24) as the form

$$
G=C_{1} e^{m y}+C_{2} e^{-m y} .
$$

Equation (24) must have exponential solutions in order that $G$ will describe surface waves; they must become vanishingly small as $y \rightarrow \infty$ :

$$
G=C_{2} e^{-m y},
$$

where $m^{2}=\omega^{2} / f_{1}$.

Substituting from (23) in to (21) we get

$$
H=f_{6} F^{\prime \prime}+f_{7} F+f_{8} C_{2} e^{-m y},
$$

where

$$
\begin{gathered}
f_{6}=\frac{f_{2}}{f_{3}\left(1-i \omega c \vartheta_{0}\right)}, \\
f_{7}=\frac{\omega^{2}\left(c^{2} f_{5}-1\right)+f_{5} \Omega^{2}}{f_{3}\left(1-i \omega c \vartheta_{0}\right)}, \\
f_{8}=\frac{i \omega\left(f_{4}-2 c f_{5} \Omega\right)}{f_{3}\left(1-i \omega c \vartheta_{0}\right)} .
\end{gathered}
$$

From (26) and (27), we can obtain the temperature $T$ and the potential function $\Psi$ as follows:

$$
\begin{gathered}
\Psi=C_{2} e^{-m y} e^{i \omega(x-c t)}, \\
T=\left[f_{6} F^{\prime \prime}+f_{7} F+f_{8} C_{2} e^{-m y}\right] e^{i \omega(x-c t)} .
\end{gathered}
$$

Substituting from (29) and (14c) into (4) for $T, \Psi, u$, and $v$ we get

$$
F^{\prime \prime \prime \prime}+a F^{\prime \prime}+b F=d C_{2} e^{-m y},
$$

where

$$
\begin{aligned}
& a=\frac{f_{10}}{f_{9}}, \quad b=\frac{f_{11}}{f_{9}}, \quad d=\frac{f_{12}}{f_{9}} \\
& f_{9}=\left(n^{*}-i \omega c t_{1}\right) k_{2}^{*} f_{6}, \\
& f_{10}=-\omega^{2} k_{1}^{*} f_{6}\left(n^{*}-i \omega c t_{1}\right)+\left(n^{*}-i \omega c t_{1}\right) k_{2}^{*} f_{7} \\
& -\rho C^{*}\left(-i \omega c n_{1}-\omega^{2} c^{2} \tau_{0}\right) f_{6} \\
& -T_{0} \beta_{2}\left(-i \omega c n_{1}-\omega^{2} c^{2} \tau_{0} n_{0}\right), \\
& f_{11}=-\omega^{2} k_{1}^{*}\left(n^{*}-i \omega c t_{1}\right) f_{7} \\
& -\rho C^{*}\left(-i \omega c n_{1}-\omega^{2} c^{2} \tau_{0}\right) f_{7} \\
& +\omega^{2} T_{0} \beta_{1}\left(-i \omega c n_{1}-\omega^{2} c^{2} \tau_{0} n_{0}\right), \\
& f_{12}=-\omega^{2} k_{1}^{*}\left(n^{*}-i \omega c t_{1}\right) f_{8} \\
& +k_{2}^{*}\left(n^{*}-i \omega c t_{1}\right) m^{2} f_{8} \\
& -\rho C^{*}\left(-i \omega c n_{1}-\omega^{2} c^{2} \tau_{0}\right) f_{8} \\
& -T_{0}\left(-i \omega c n_{1}-\omega^{2} c^{2} \tau_{0} n_{0}\right) \\
& \times\left(-i \omega m \beta_{1}+i \omega m \beta_{2}\right) .
\end{aligned}
$$

The solution of (30) is

$$
F=C_{4} e^{-f_{14} y}+C_{6} e^{-f_{16} y}+M C_{2} e^{-m y},
$$

where

$$
\begin{gathered}
f_{14}=\sqrt{\frac{-a \sqrt{a^{2}-4 b}}{2},} \\
f_{16}=\sqrt{-\frac{a}{2}+\frac{1}{2} \sqrt{a^{2}-4 b}}, \\
M=\frac{d}{m^{4}+a m^{2}-b m} .
\end{gathered}
$$

After finding the values of $F$ we get the following values:

$$
\begin{gathered}
\varphi=\left[C_{4} e^{-f_{14} y}+C_{6} e^{-f_{16} y}+M C_{2} e^{-m y}\right] e^{i \omega(x-c t)}, \\
\Psi=C_{2} e^{-m y} e^{i \omega(x-c t)}, \\
T=\left\{\left(f_{6} f_{14}^{2}+f_{7}\right) C_{4} e^{-f_{14} y}+\left(f_{6} f_{16}^{2}+f_{7}\right) C_{6} e^{-f^{\prime}{ }_{16} y}\right. \\
\left.+\left[\left(m^{2} f_{6}+f_{7}\right) M+f_{8}\right] C_{2} e^{-m y}\right\} e^{i \omega(x-c t)} .
\end{gathered}
$$

Substituting from (34), (35), and (36) into (14c), we get the components of displacement as

$$
\begin{aligned}
u=\{ & \left\{\omega C_{4} e^{-f_{14} y}+i \omega C_{6} e^{-f_{16} y}\right. \\
& \left.+(i \omega M-m) C_{2} e^{-m y}\right\} e^{i \omega(x-c t)},
\end{aligned}
$$




$$
\begin{aligned}
v=\{ & -f_{14} C_{4} e^{-f_{14} y}-f_{16} C_{6} e^{-f_{16} y} \\
& \left.-(m M+i \omega) C_{2} e^{-m y}\right\} e^{i \omega(x-c t)} .
\end{aligned}
$$

Substituting from (36), (37), and (38a) in to (9)-(12) we obtain

$$
\begin{aligned}
\tau_{11}=\left\{\lambda\left(f_{14}^{2}-\omega^{2}\right)+\alpha\left(f_{14}^{2}-2 \omega^{2}\right)-4 \mu_{L} \omega^{2}\right. \\
\left.+2 \mu_{T} \omega^{2}-\beta \omega^{2}-\beta_{1}\left(1-i \omega \vartheta_{0}\right)\left(f_{6}+f_{7}\right)\right\} \\
\times C_{4} e^{-f_{14} y} e^{i \omega(x-c t)}+\left\{\lambda\left(f_{16}^{2}-\omega^{2}\right)+\alpha\left(f_{16}^{2}-2 \omega^{2}\right)\right. \\
-4 \mu_{L} \omega^{2}+2 \mu_{T} \omega^{2}-\beta \omega^{2} \\
\left.-\beta_{1}\left(1-i \omega \vartheta_{0}\right)\left(f_{6} f_{16}^{2}+f_{7}\right)\right\}
\end{aligned}
$$$$
\times C_{6} e^{-f_{16} y} e^{i \omega(x-c t)}
$$$$
+\left\{\lambda\left(m^{2}-\omega^{2}\right)+\alpha\left[\left(m^{2}-2 \omega^{2}\right) M-i \omega m\right]\right.
$$$$
-\left(4 \mu_{L}-2 \mu_{T}+\beta\right)\left(\omega^{2} M+i \omega m\right)
$$$$
\left.-\beta_{1}\left(1-i \omega \vartheta_{0}\right)\left[\left(m^{2} f_{6}+f_{7}\right) M+f_{8}\right]\right\}
$$$$
\times C_{2} e^{-m y} e^{i \omega(x-c t)} \text {, }
$$$$
\tau_{22}=\left\{\lambda\left(f_{14}^{2}-\omega^{2}\right)-\alpha \omega^{2}+2 \mu_{T} f_{14}^{2}\right.
$$$$
-\beta_{2}\left(1-i \omega c \vartheta_{0}\right)
$$$$
\left.\times\left(f_{6} f_{14}{ }^{2}+f_{7}\right)\right\} C_{4} e^{-f_{14} y} e^{i \omega(x-c t)}
$$$$
+\left\{\lambda\left(f_{16}{ }^{2}-\omega^{2}\right)-\alpha \omega^{2}+2 \mu_{T} f_{16}^{2}\right.
$$$$
\left.-\beta_{2}\left(1-i \omega c \vartheta_{0}\right)\left(f_{6} f_{16}^{2}+f_{7}\right)\right\}
$$$$
\times C_{6} e^{-f_{16} y} e^{i \omega(x-c t)}
$$$$
+\left\{\lambda\left(m^{2}-\omega^{2}\right) M-\alpha\left(\omega^{2} M+i \omega m\right)\right.
$$$$
+2 \mu_{T} m(m M+i \omega)
$$$$
\left.-\beta_{2}\left(1-i \omega c \vartheta_{0}\right)\left[\left(m^{2} f_{6}+f_{7}\right) M+f_{8}\right]\right\}
$$$$
\times C_{2} e^{-m y} e^{i \omega(x-c t)}
$$

$$
\begin{aligned}
\tau_{12}=\{[ & \left.-2 i \omega \mu_{L} f_{14}\right] e^{-f_{14} y} C_{4}+\left[-2 i \omega \mu_{L} f_{16}\right] C_{6} e^{-f_{16} y} \\
+ & {\left.\left[\mu_{L}\left(-2 i \omega m M+\omega^{2}-m^{2}\right)\right] C_{2} e^{-m y}\right\} e^{i \omega(x-c t)} . }
\end{aligned}
$$

\section{Boundary Conditions}

The stresses components, the displacement components, and temperature at the boundary surface between the media $M_{1}$ and $M_{2}$ must be continuous at all times and positions.

Consider the following:

$$
\begin{array}{ll}
\tau_{22}=\tau_{22}^{\prime} & \text { at } y=0, \\
\tau_{12}=\tau_{12}^{\prime} & \text { at } y=0,
\end{array}
$$

$$
\begin{gathered}
u=u^{\prime} \quad \text { at } y=0, \\
v=v^{\prime} \quad \text { at } y=0, \\
\frac{\partial T}{\partial y}+h T=\frac{\partial T^{\prime}}{\partial y}+h^{\prime} T^{\prime} \quad \text { at } y=0 .
\end{gathered}
$$

Finally, eliminating the constants $C_{2}, C_{4}, C_{6}, C_{2}^{\prime}, C_{4}^{\prime}$, and $C_{6}^{\prime}$ from (10), (12), (36), (37), and (38a), (38b), (38c), and (38d), we get

$$
\operatorname{det}\left(a_{i j}\right)=0, \quad i, j=1,2,3,
$$

where

$$
\begin{aligned}
& a_{11}=\lambda\left(f_{14}^{2}-\omega^{2}\right)-\alpha \omega^{2}+2 \mu_{T} f_{14}^{2} \\
& -\beta_{2}\left(1-i \omega c \vartheta_{0}\right)\left(f_{6} f_{14}^{2}+f_{7}\right), \\
& a_{12}=\lambda\left(f_{16}^{2}-\omega^{2}\right)-\alpha \omega^{2}+2 \mu_{T} f_{16}^{2} \\
& -\beta_{2}\left(1-i \omega c \vartheta_{0}\right)\left(f_{6} f_{16}^{2}+f_{7}\right), \\
& a_{13}=\lambda\left(m^{2}-\omega^{2}\right) M-\alpha\left(\omega^{2} M+i \omega m\right) \\
& +2 \mu_{T} m(m M+i \omega)-\beta_{2}\left(1-i \omega c \vartheta_{0}\right) \\
& \times\left[\left(m^{2} f_{6}+f_{7}\right) M+f_{8}\right], \\
& a_{14}=-\left[\lambda^{\prime}\left(f_{14}^{\prime 2}-\omega^{2}\right)-\alpha^{\prime} \omega^{2}+2 \mu_{T}^{\prime} f_{14}^{\prime 2}\right. \\
& \left.-\beta_{2}^{\prime}\left(1-i \omega c \vartheta_{0}\right)\left(f_{6}^{\prime} f_{14}^{\prime 2}+f_{7}^{\prime}\right)\right], \\
& a_{15}=\left[\lambda^{\prime}\left(f_{16}^{\prime 2}-\omega^{2}\right)-\alpha^{\prime} \omega^{2}+2 \mu_{T}^{\prime} f_{16}^{\prime 2}\right. \\
& \left.-\beta_{2}^{\prime}\left(1-i \omega c \vartheta_{0}\right)\left(f_{6}^{\prime}{f_{16}^{\prime}}^{2}+f_{7}^{\prime}\right)\right], \\
& a_{16}=-\left[\lambda^{\prime}\left(m^{\prime 2}-\omega^{2}\right) M^{\prime}-\alpha^{\prime}\left(\omega^{2} M^{\prime}+i \omega m^{\prime}\right)\right. \\
& +2 \mu_{T}^{\prime} m^{\prime}\left(m^{\prime} M^{\prime}+i \omega\right) \\
& -\beta_{2}^{\prime}\left(1-i \omega c \vartheta_{0}\right) \\
& \left.\times\left[\left(m^{\prime 2} f_{6}^{\prime}+f_{7}^{\prime}\right) M^{\prime}+f_{8}^{\prime}\right]\right], \\
& a_{21}=-2 i \omega \mu_{L} f_{14}, \quad a_{22}=-2 i \omega \mu_{L} f_{16} \text {, } \\
& a_{23}=\mu_{L}\left(-2 i \omega m M+\omega^{2}-m^{2}\right), \\
& a_{24}=2 i \omega \mu_{L}^{\prime} f_{14}^{\prime}, \\
& a_{25}=2 i \omega \mu_{L}^{\prime} f_{16}^{\prime}, \\
& a_{26}=-\left[\mu_{L}^{\prime}\left(-2 i \omega m^{\prime} M^{\prime}+\omega^{2}-m^{\prime 2}\right)\right] \text {, } \\
& a_{31}=i \omega, \quad a_{32}=i \omega, \quad a_{33}=i \omega M-m, \\
& a_{34}=-i \omega, \quad a_{35}=-i \omega, \quad a_{36}=-\left(i \omega M^{\prime}-m^{\prime}\right) \text {, }
\end{aligned}
$$




$$
\begin{gathered}
a_{41}=-f_{14}, \quad a_{42}=-f_{16}, \quad a_{43}=-(m M+i \omega), \\
a_{44}=f_{14}^{\prime}, \quad a_{45}=f_{16}^{\prime}, \quad a_{46}=m^{\prime} M+i \omega, \\
a_{51}=\left(f_{6} f_{14}^{2}+f_{7}\right)\left(h-f_{14}\right), \\
a_{52}=\left(f_{6} f_{16}^{2}+f_{7}\right)\left(h-f_{16}\right), \\
a_{53}=\left[\left(m^{2} f_{6}+f_{7}\right) M+f_{8}\right](1-m), \\
a_{54}=-\left[\left(f_{6}^{\prime} f_{14}^{\prime 2}+f_{7}^{\prime}\right)\left(h^{\prime}-f_{14}^{\prime}\right)\right], \\
a_{55}=-\left[\left(f_{6}^{\prime} f_{16}^{\prime \prime 2}+f_{7}^{\prime}\right)\left(h^{\prime}-f_{16}^{\prime}\right)\right], \\
a_{56}=-\left[\left(m^{\prime 2} f_{6}^{\prime}+f_{7}^{\prime}\right) M^{\prime}+f_{8}^{\prime}\right]\left(1-m^{\prime}\right), \\
a_{61}=\left(f_{6} f_{14}{ }^{2}+f_{7}\right), \\
a_{63}=\left[\left(m^{2} f_{6}+f_{7}\right) M+f_{8}\right], \\
a_{64}=-\left(f_{6}^{\prime} f_{14}^{\prime 2}+f_{7}^{\prime}\right), \\
a_{65}=-\left(f_{6}^{\prime} f_{16}^{\prime 2}+f_{7}^{\prime}\right), \\
a_{66}=-\left[\left(m^{\prime 2} f_{6}^{\prime}+f_{7}^{\prime}\right) M^{\prime}+f_{8}^{\prime}\right] .
\end{gathered}
$$

\section{Stoneley Waves}

It is the generalized form of Rayleigh waves in which we assume that the waves are propagated along the common boundary of two semi-infinite media $M_{1}$ and $M_{2}$. Therefore (40) determines the wave velocity equation for Stoneley waves in anisotropic fibre-reinforced solid thermoelastic media under the influence of gravity. Clearly, from (40), it follows that wave velocity of the Stoneley waves depends upon the parameters for fibre-reinforced of the material medium, gravity, rotation, and the densities of both mediums, since the wave velocity equation (40) for Stoneley waves under the present circumstances depends on the particular value of $\omega$ and creates a dispersion of a general wave form. Further, (40), of course, is in complete agreement with the corresponding classical result, when the effect of gravity, rotation, and parameters for fibre-reinforcement is ignored.

\section{Rayleigh Waves}

To investigate the possibility of Rayleigh waves in anisotropic fibre-reinforced thermoelastic media, we replace medium $M_{2}$ by vacuum, in the preceding problem, since the boundary $(y=0)$ is adjacent to vacuum. So the boundary condition in this case may be expressed as

$$
\begin{gathered}
\tau_{12}=0 \quad \text { at } y=0, \\
v=0 \quad \text { at } y=0, \\
\frac{\partial T}{\partial y}+h T=0 \quad \text { at } y=0 .
\end{gathered}
$$

Eliminating the constants $C_{2}, C_{4}$, and $C_{6}$ from (10), (12), (34), (35), (36), and (37), we get

$$
\operatorname{det}\left(b_{i, j}\right)=0, \quad i, j=1,2,3 \text {, }
$$

where

$$
\begin{gathered}
b_{11}=-2 i \omega \mu_{L} f_{14}, \quad b_{12}=-2 i \omega \mu_{L} f_{16}, \\
b_{13}=\mu_{L}\left(-2 i \omega m M+\omega^{2}+m^{2}\right), \quad b_{21}=f_{14}, \\
b_{22}=f_{16}, \quad b_{23}=(m M+i \omega), \\
b_{31}=\left(h-f_{14}\right)\left(f_{6} f_{14}^{2}+f_{7}\right), \\
b_{32}=\left(h-f_{16}\right)\left(f_{6} f_{16}^{2}+f_{7}\right), \\
b_{33}=(h-m)\left[\left(m^{2} f_{6}+f_{7}\right) M+f_{8}\right] .
\end{gathered}
$$

\section{Love Waves}

To investigate the possibility of love waves in a fibrereinforced thermoelastic solid media, we replace medium $M_{2}$ that is obtained by two horizontal plane surfaces at a distance $H$-apart, while $M_{1}$ remains infinite. For medium $M_{1}$, the displacement component $v$ remains the same as in general case given by (38a), (38b), (38c), and (38d).

The boundary condition of Love wave is as follows:

$$
\begin{gathered}
\tau_{12}=\tau_{12}^{\prime}, \quad \text { at } y=0, \\
v=v^{\prime}, \quad \text { at } y=0, \\
T=T^{\prime}, \quad \text { at } y=0, \\
\tau_{12}^{\prime}=0, \quad \text { at } y=-H, \\
v^{\prime}=0 \quad \text { at } y=-H, \quad T^{\prime}=0 \quad \text { at } y=-H .
\end{gathered}
$$

Eliminating the constants $C_{2}, C_{4}, C_{6}, C_{2}^{\prime}, C_{4}^{\prime}$, and $C_{6}^{\prime}$ from (10), (12), (34), (35), (36), and (37), we get

$$
\begin{gathered}
\operatorname{det}\left(c_{i, j}\right)=0, \quad i, j=1,2,3, \\
c_{11}=-2 i \omega \mu_{L} f_{14}, \quad c_{12}=-2 i \omega \mu_{L} f_{16}, \\
c_{13}=\mu_{L}\left(-2 i \omega m M+\omega^{2}+m^{2}\right), \\
c_{14}=2 i \omega \mu_{L}^{\prime} f_{14}^{\prime}, \quad c_{15}=2 i \omega \mu_{L}^{\prime} f_{16}^{\prime}, \\
c_{16}=-\mu_{L}^{\prime}\left(-2 i \omega m^{\prime} M^{\prime}+\omega^{2}+m^{\prime 2}\right), \\
c_{21}=f_{14}, \quad c_{22}=f_{16}, \\
c_{23}=m M+i \omega, \quad c_{24}=-f_{14}^{\prime} \\
c_{25}=-f_{16}^{\prime}, \quad c_{26}=-\left(m^{\prime} M^{\prime}+i \omega\right), \\
c_{31}=f_{6} f_{14}^{2}+f_{7}, \quad c_{32}=f_{6} f_{16}^{2}+f_{7}, \\
c_{33}=\left(m^{2} f_{6}+f_{7}\right) M+f_{8}, \\
c_{34}=-\left(f_{6}^{\prime} f_{14}^{\prime 2}+f_{7}^{\prime}\right), \quad c_{35}=-\left(f_{6}^{\prime} f_{16}^{\prime 2}+f^{\prime}\right), \\
c_{36}=-\left[\left(m^{\prime 2} f_{6}^{\prime}+f_{7}^{\prime}\right) M^{\prime}+f_{8}^{\prime}\right],
\end{gathered}
$$



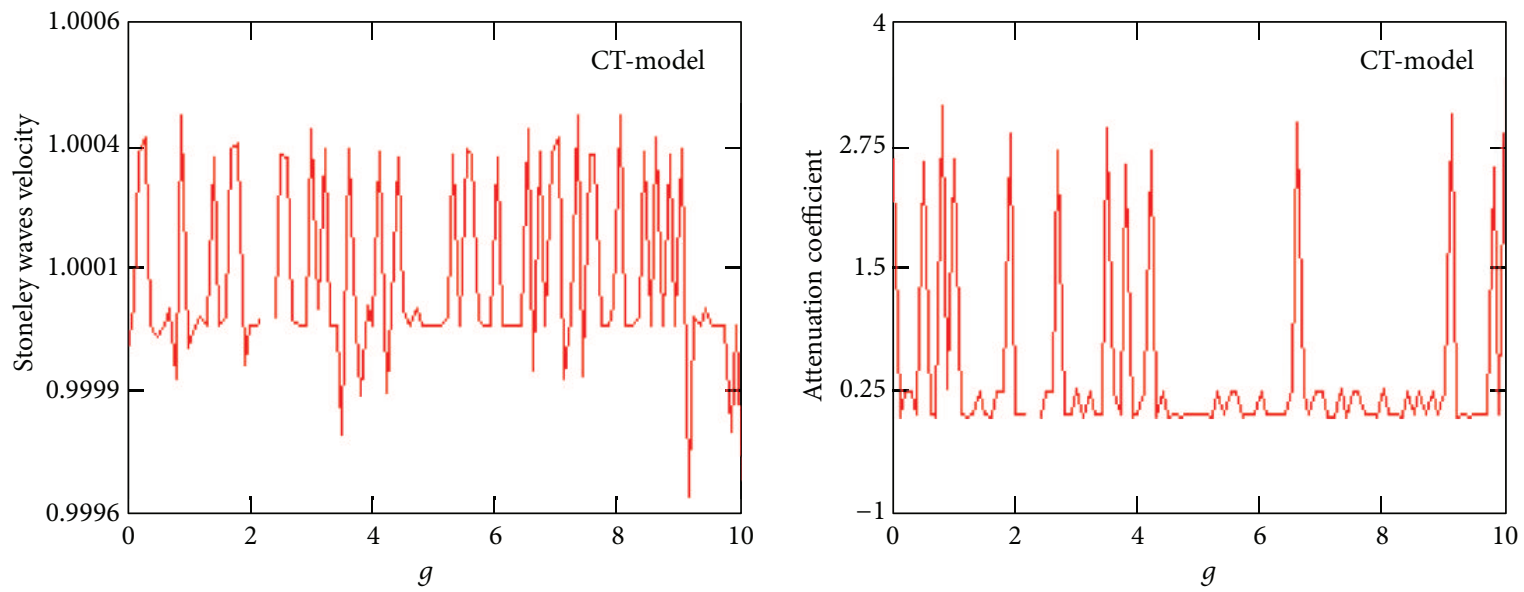

FIGURE 1: Variation of the Stoneley waves velocity and attenuation coefficient (CT) model with respect to the gravity.
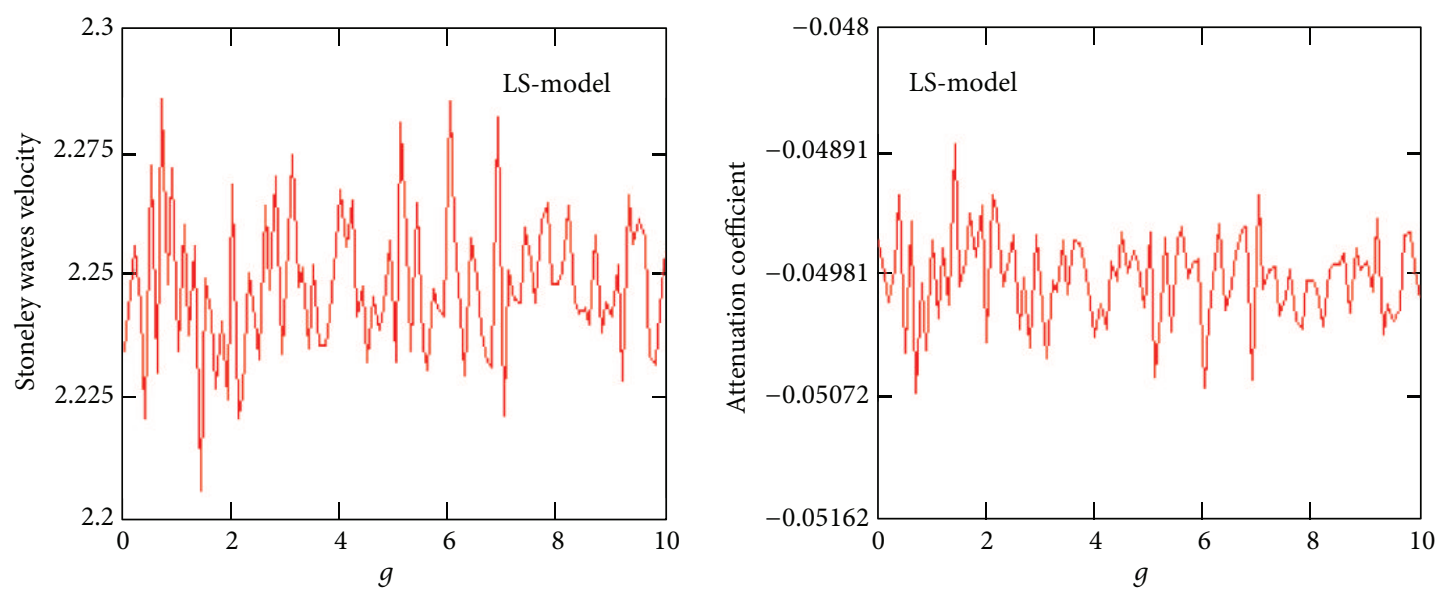

FIGURE 2: Variation of the Stoneley waves velocity and attenuation coefficient (LS) model with respect to the gravity.
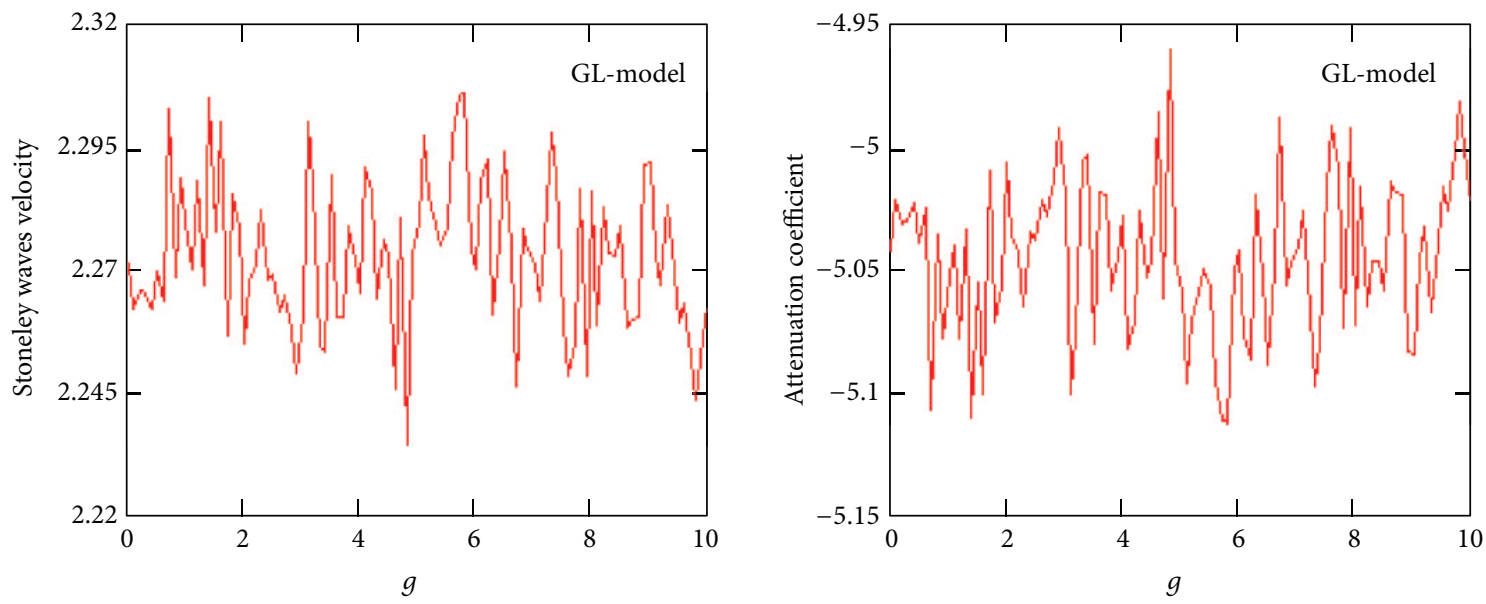

FIGURE 3: Variation of the Stoneley waves velocity and attenuation coefficient (GL) model with respect to the gravity. 

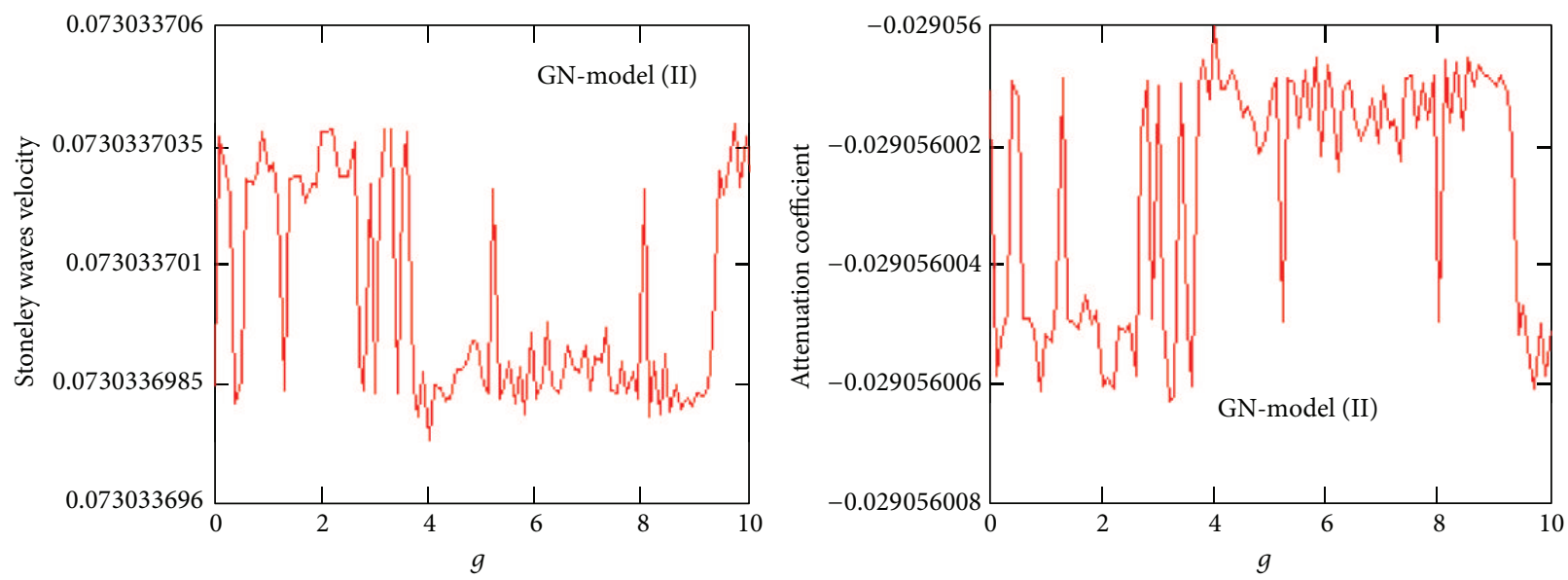

FIGURE 4: Variation of the Stoneley waves velocity and attenuation coefficient (GN type (II)) model with respect to the gravity.
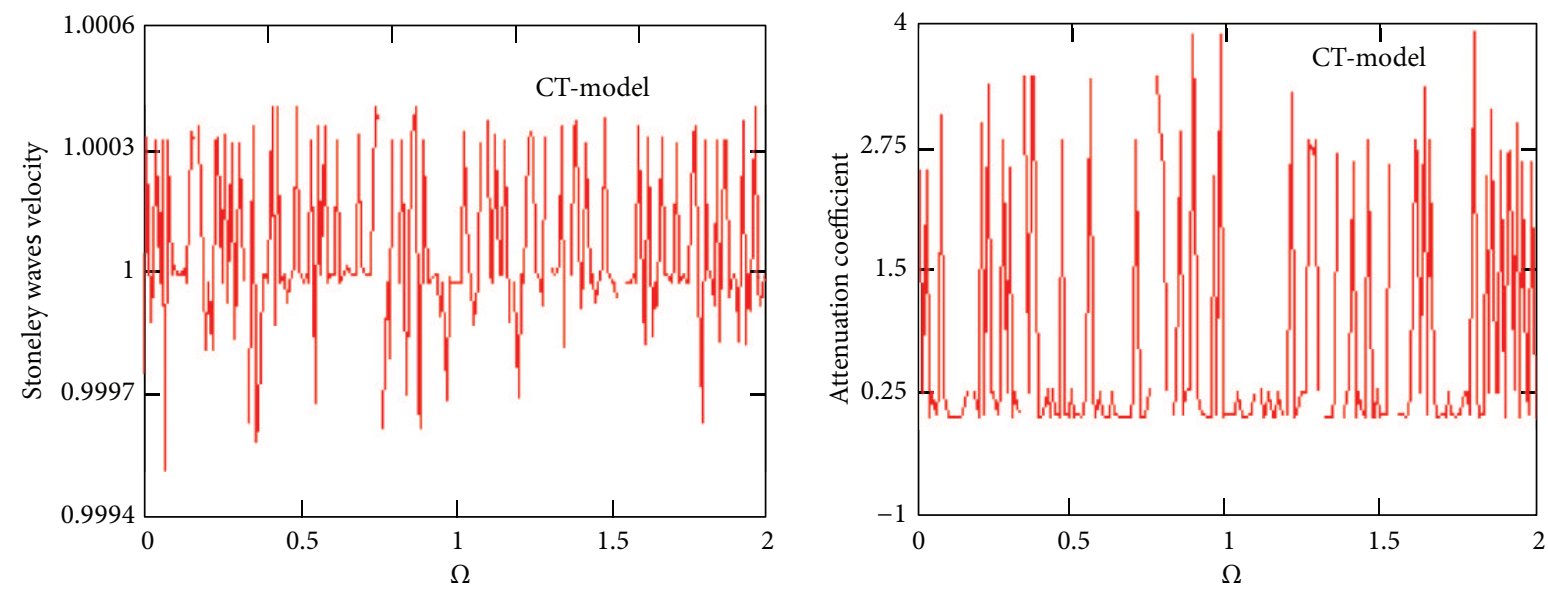

FIGURE 5: Variation of the Stoneley waves velocity and attenuation coefficient (CT) model with respect to the rotation.
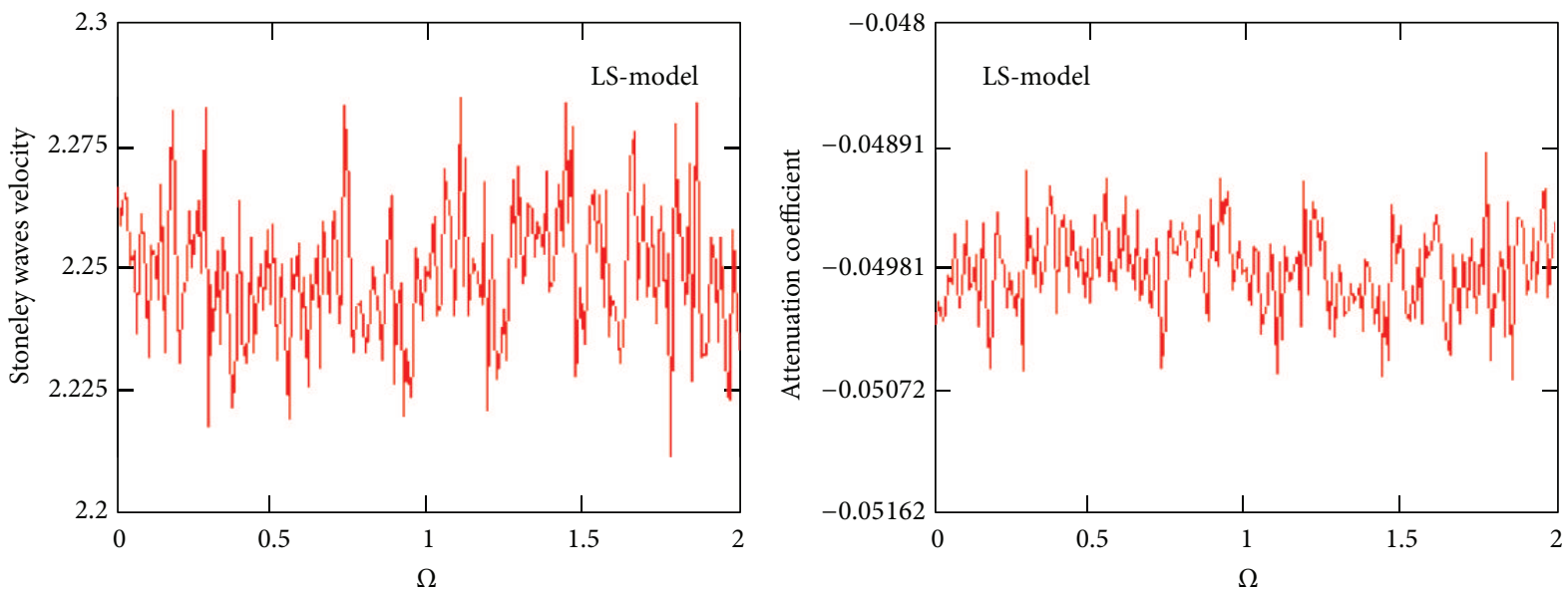

FIGURE 6: Variation of the Stoneley waves velocity and attenuation coefficient (LS) model with respect to the rotation. 

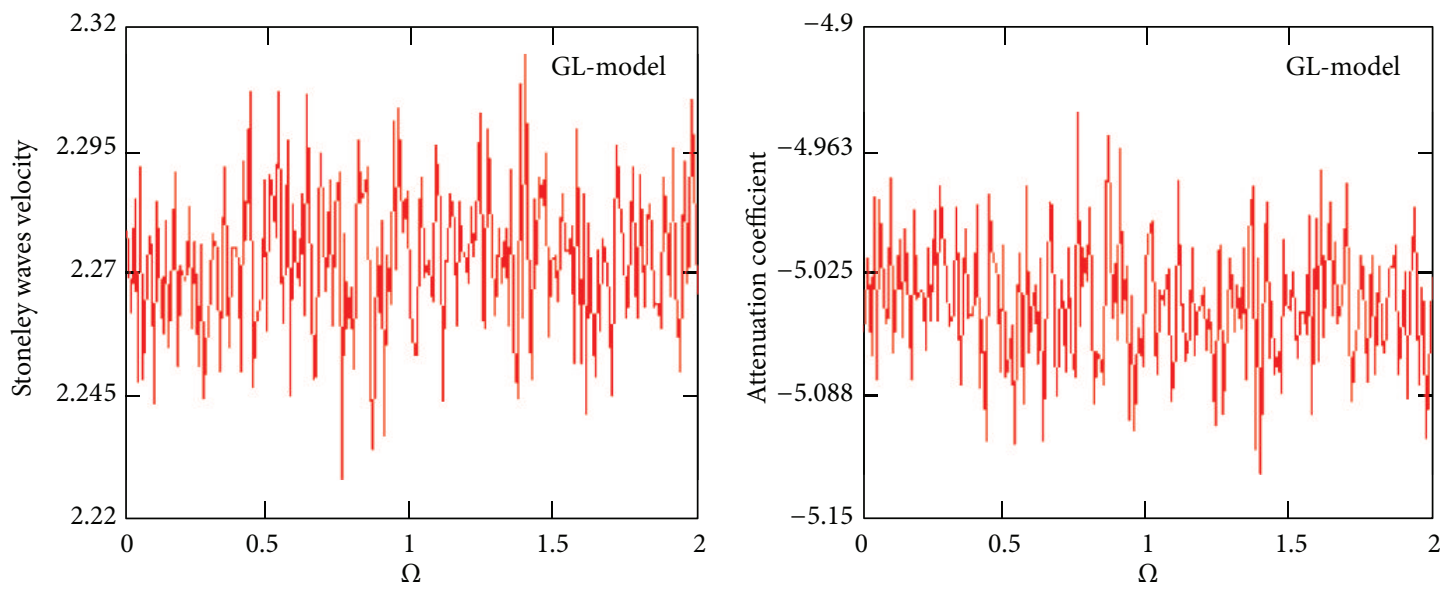

FIGURE 7: Variation of the Stoneley waves velocity and attenuation coefficient (GL) model with respect to the rotation.
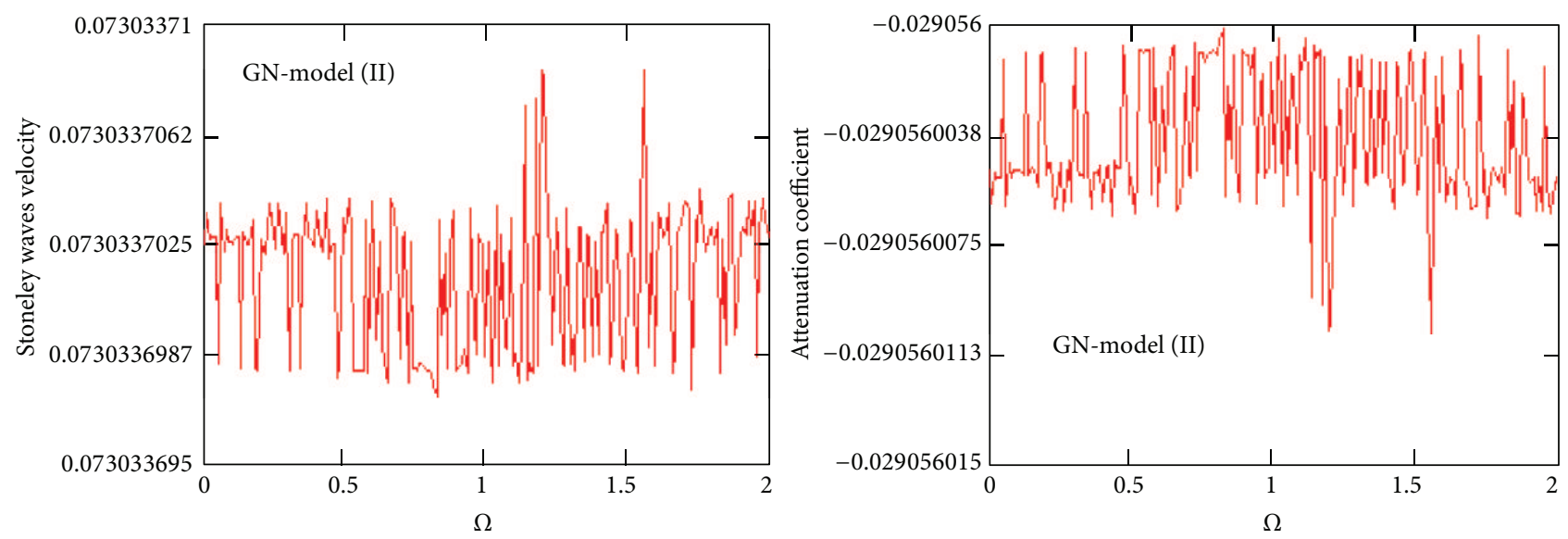

FIGURE 8: Variation of the Stoneley waves velocity and attenuation coefficient (GN type (II)) model with respect to the rotation.

$$
\begin{gathered}
c_{44}=2 i \omega \mu_{L}^{\prime} f_{14}^{\prime} e^{f_{14}^{\prime} H}, \\
c_{45}=2 i \omega \mu_{L}^{\prime} f_{16}^{\prime} e^{f_{16}^{\prime} H}, \\
c_{46}=-\mu_{L}^{\prime}\left(-2 i \omega m^{\prime} M^{\prime}+\omega^{2}+m^{\prime 2}\right) e^{m^{\prime} H}, \\
a_{51}=0, \quad c_{52}=0, \quad c_{53}=0, \quad c_{54}=f_{14}^{\prime} e^{f_{14}^{\prime} H}, \\
c_{55}=f_{16}^{\prime} e^{f_{16}^{\prime} H}, \quad c_{56}=\left(m^{\prime} M^{\prime}+i \omega\right) e^{m^{\prime} H}, \\
c_{61}=0, \quad c_{62}=0, \quad c_{63}=0, \\
c_{64}=\left(f_{6}^{\prime} f_{14}^{\prime 2}+f_{7}^{\prime}\right) e^{f_{14}^{\prime} H}, \\
c_{65}=\left(f_{6}^{\prime} f_{16}^{\prime 2}+f_{7}^{\prime}\right) e^{f_{16}^{\prime} H}, \\
c_{66}=\left[\left(m^{\prime 2} f_{6}^{\prime}+f_{7}^{\prime}\right) M^{\prime}+f_{8}^{\prime}\right] e^{m^{\prime} H} .
\end{gathered}
$$

\section{Numerical Results and Discussion}

With a view to illustrating the analytical procedure presented earlier, we now consider a numerical example for which computational results are given. The results depict the variation of
Stoneley waves velocity and attenuation coefficient, Stoneley secular equation, Rayleigh secular equation, Rayleigh waves velocity and attenuation coefficient, Love waves velocity and attenuation coefficient, and Love secular equation in the context of four theories. To study the influence of gravity field and rotation with reinforcement on wave propagation, we now present some numerical results for the physical constants:

$$
\begin{gathered}
\rho=2660 \mathrm{~kg} / \mathrm{m}^{3}, \quad \lambda=5.65 \times 10^{10} \mathrm{Nm}^{-2}, \\
\mu_{T}=2.46 \times 10^{10} \mathrm{Nm}^{-2}, \quad \mu_{L}=5.66 \times 10^{10} \mathrm{Nm}^{-2}, \\
C^{*}=0.787 \times 10^{3} \mathrm{JKg}^{-1} \mathrm{deg}^{-1}, \\
\alpha=-1.28 \times 10^{10} \mathrm{Nm}^{-2}, \quad \beta=220.90 \times 10^{10} \mathrm{Nm}^{-2}, \\
K_{1}^{*}=0.0921 \times 10^{3} \mathrm{Jm}^{-1} \mathrm{deg}^{-1} \mathrm{~s}^{-1}, \\
K_{2}^{*}=0.0963 \times 10^{3} \mathrm{Jm}^{-1} \mathrm{deg}^{-1} \mathrm{~s}^{-1}, \\
\alpha_{1}=0.017 \times 10^{4} \mathrm{deg}^{-1}, \\
\alpha_{2}=0.015 \times 10^{4} \mathrm{deg}^{-1}, \quad T_{0}=293^{0} \mathrm{~K} .
\end{gathered}
$$

The output is plotted in Figures 1-30. 


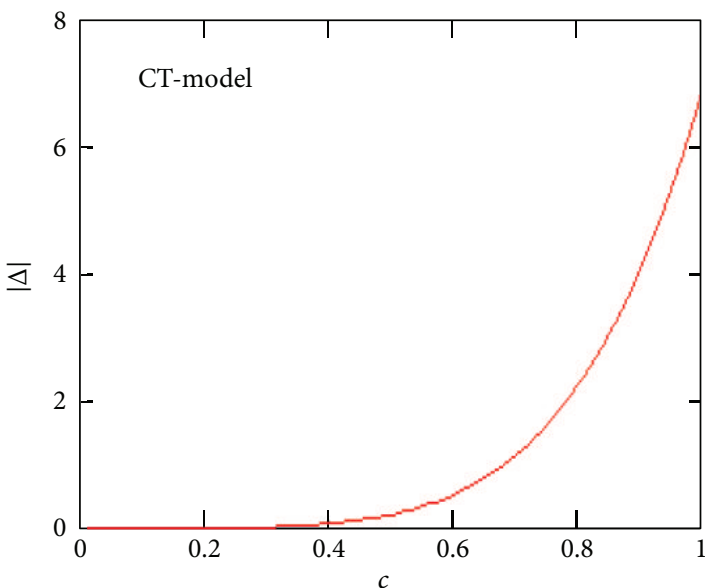

(a)

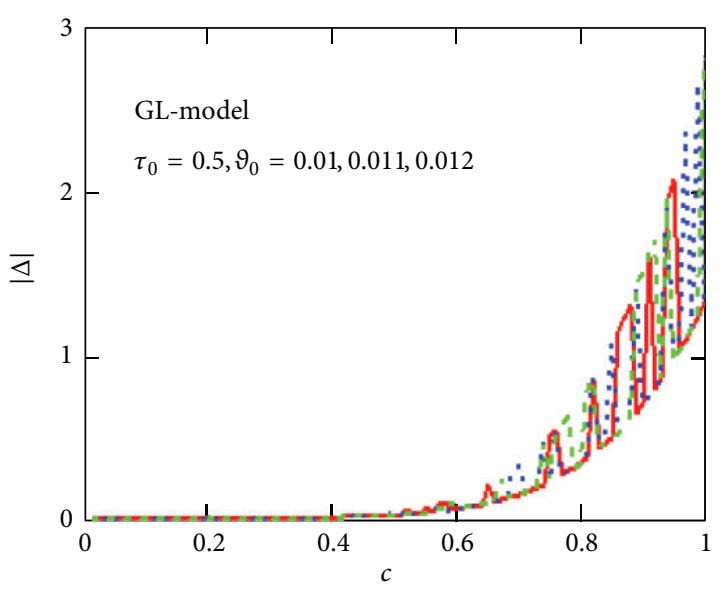

(c)

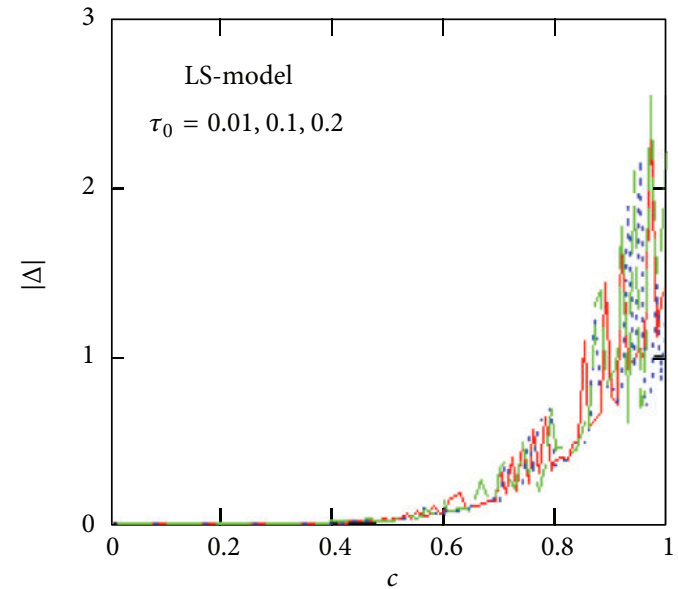

(b)

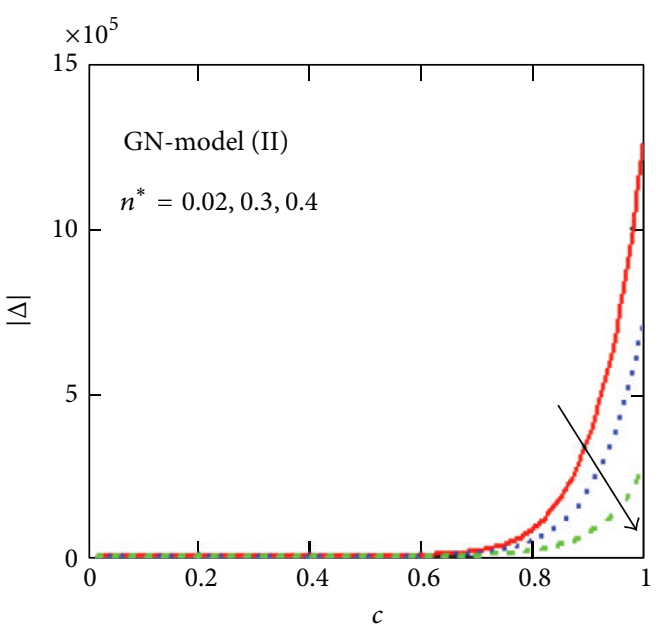

(d)

FIGURE 9: Variation of the Stoneley secular equation for the (CT, LS, GL, and GN (type (II)) models with respect to the phase velocity.
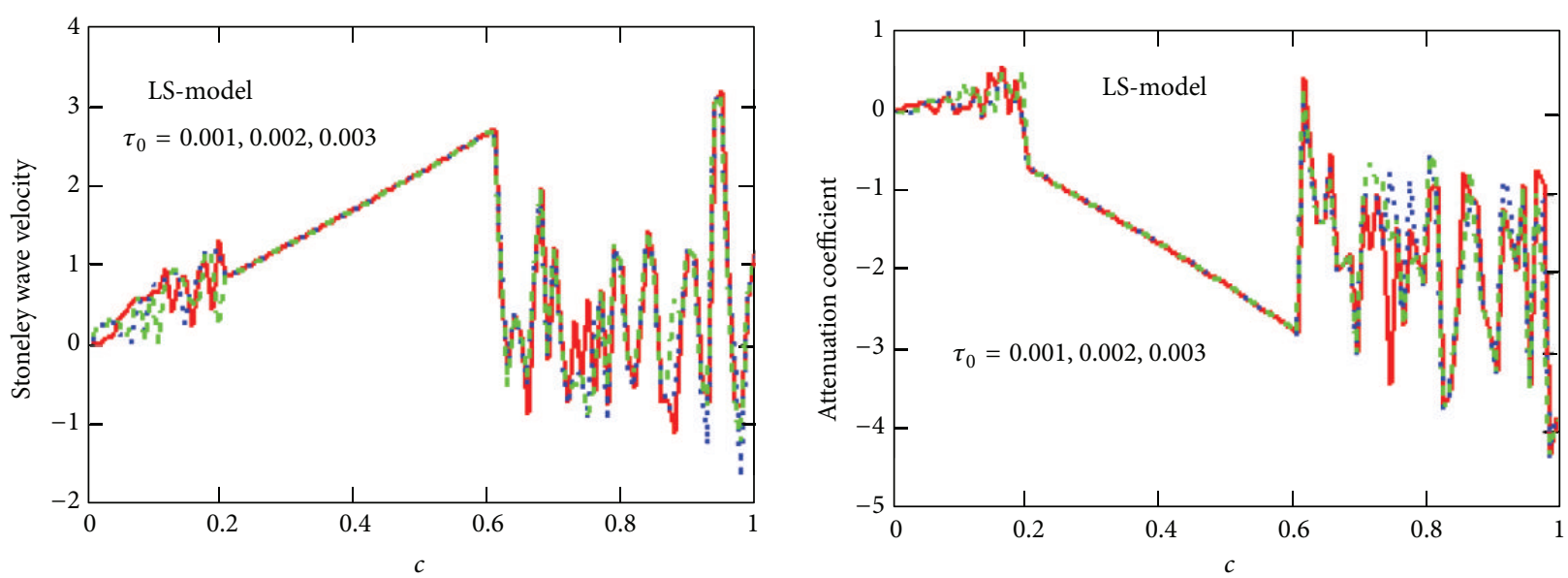

FIGURE 10: Variation of the Stoneley waves velocity and attenuation coefficient (LS) model with respect to the phase velocity. 

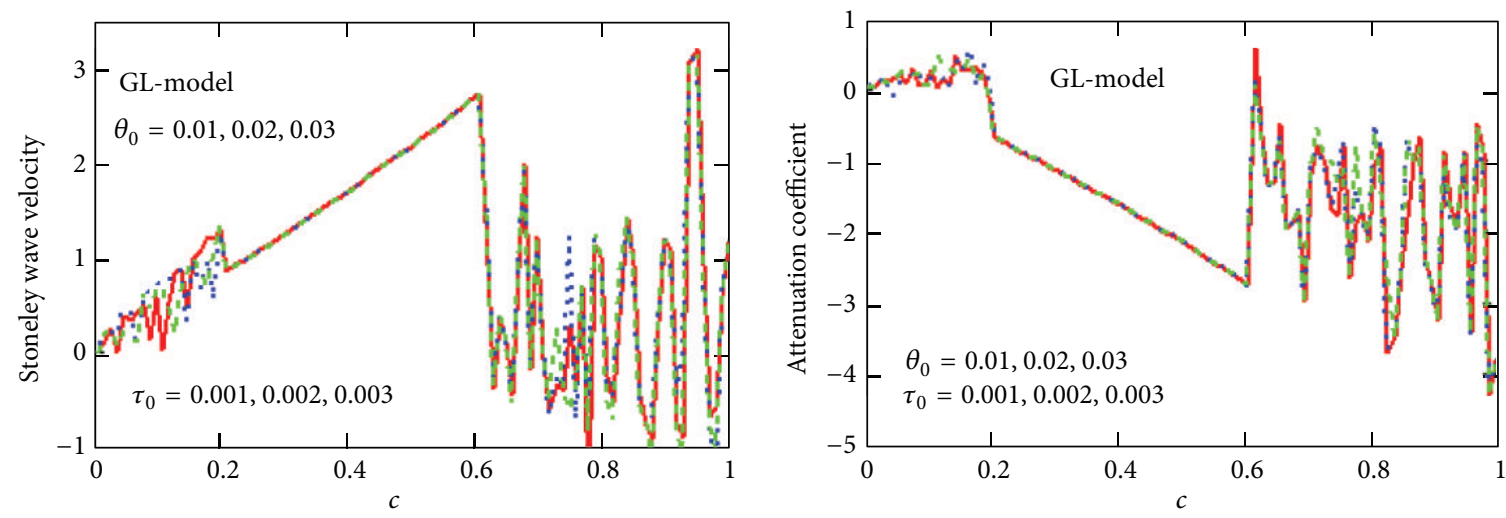

FIGURE 11: Variation of the Stoneley waves velocity and attenuation coefficient (GL) model with respect to the phase velocity.
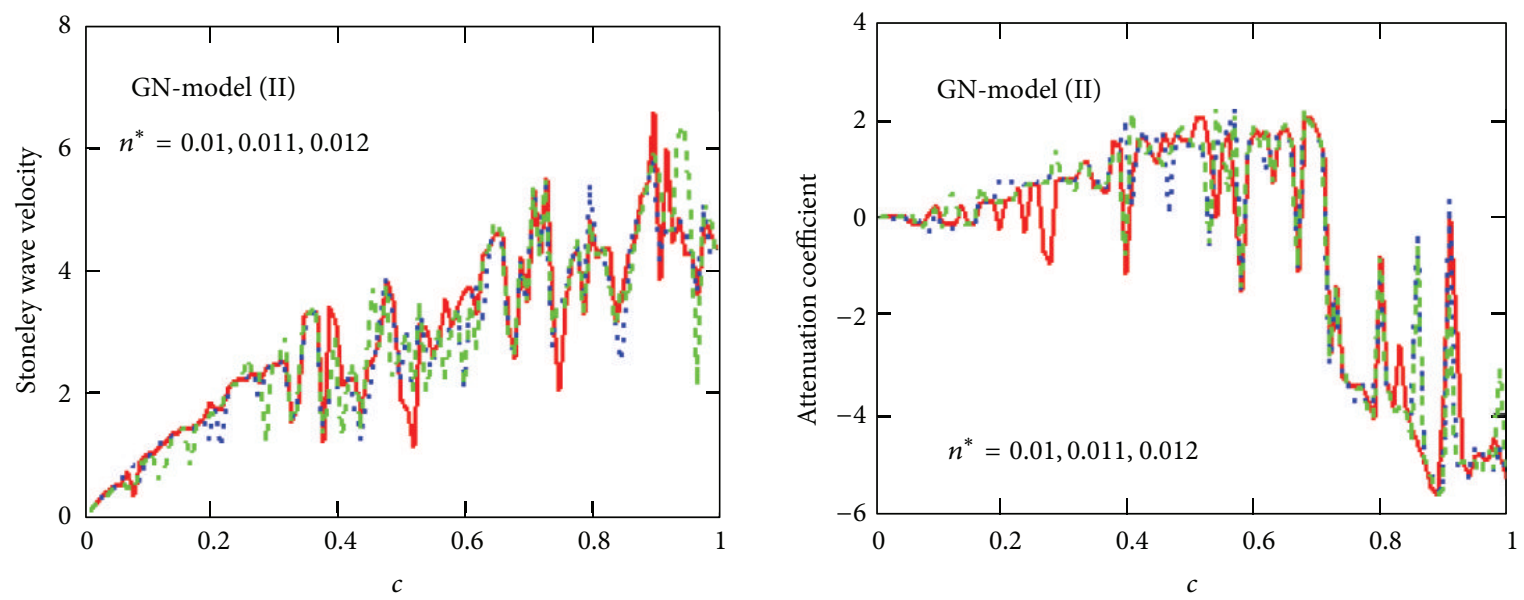

FIGURE 12: Variation of the Stoneley waves velocity and attenuation coefficient (GN type (II)) model with respect to the phase velocity.
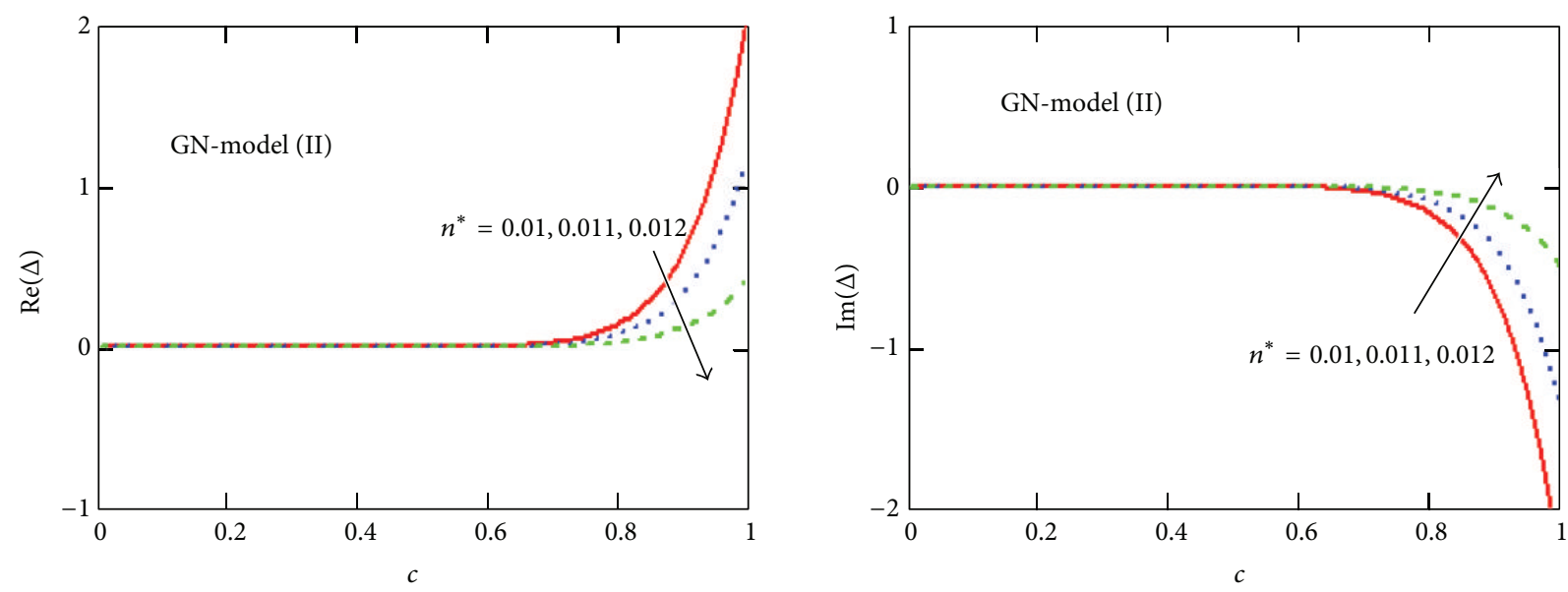

FIGURE 13: Variation of the Stoneley waves velocity and attenuation coefficient (GN type (II)) model with respect to the phase velocity.

Figures $1,2,3,4,5,6,7$, and 8 show the variation of the Stoneley waves and attenuation coefficient value with respect to gravity field $g$ and rotation $\Omega$, respectively, has oscillatory behavior in the whole range of gravity $g$ and rotation for C-T theory, L-S theory, G-L theory, and G-N theory. These figures indicate that the medium along $g$ and $\Omega$ undergoes compressive deformation due to the thermal shock. In both figureures, it is clear that the Stoneley wave velocity and attenuation coefficient have a nonzero value only in a bounded region of space. 

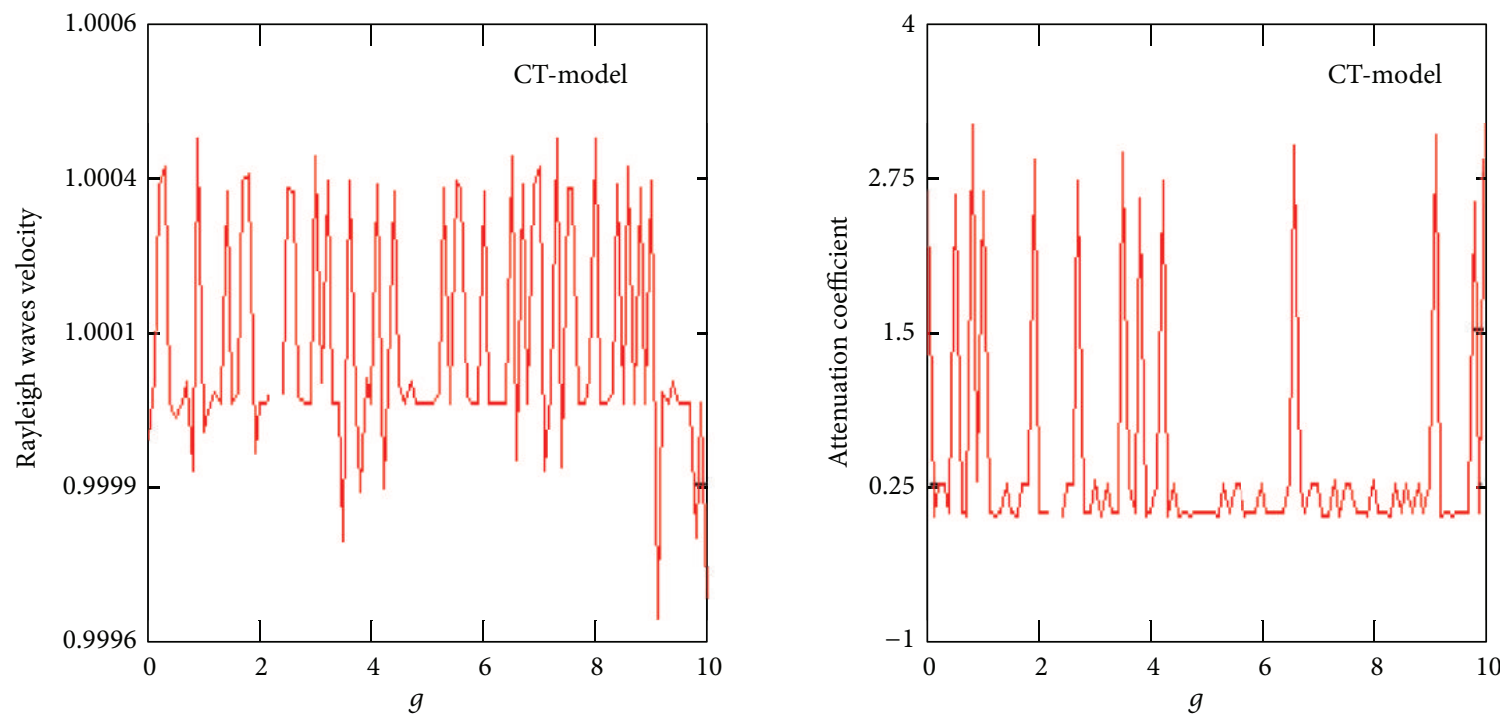

FIGURE 14: Variation of the Rayleigh waves velocity and attenuation coefficient (CT) model with respect to the gravity.
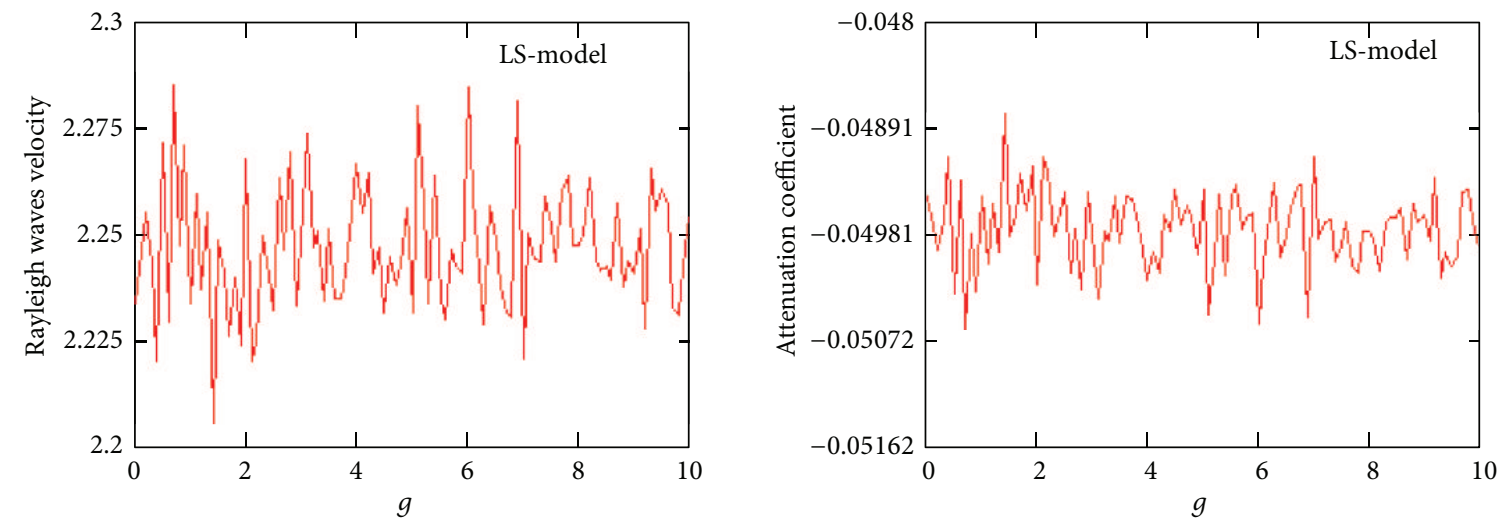

FIGURE 15: Variation of the Rayleigh waves velocity and attenuation coefficient (LS) model with respect to the gravity.
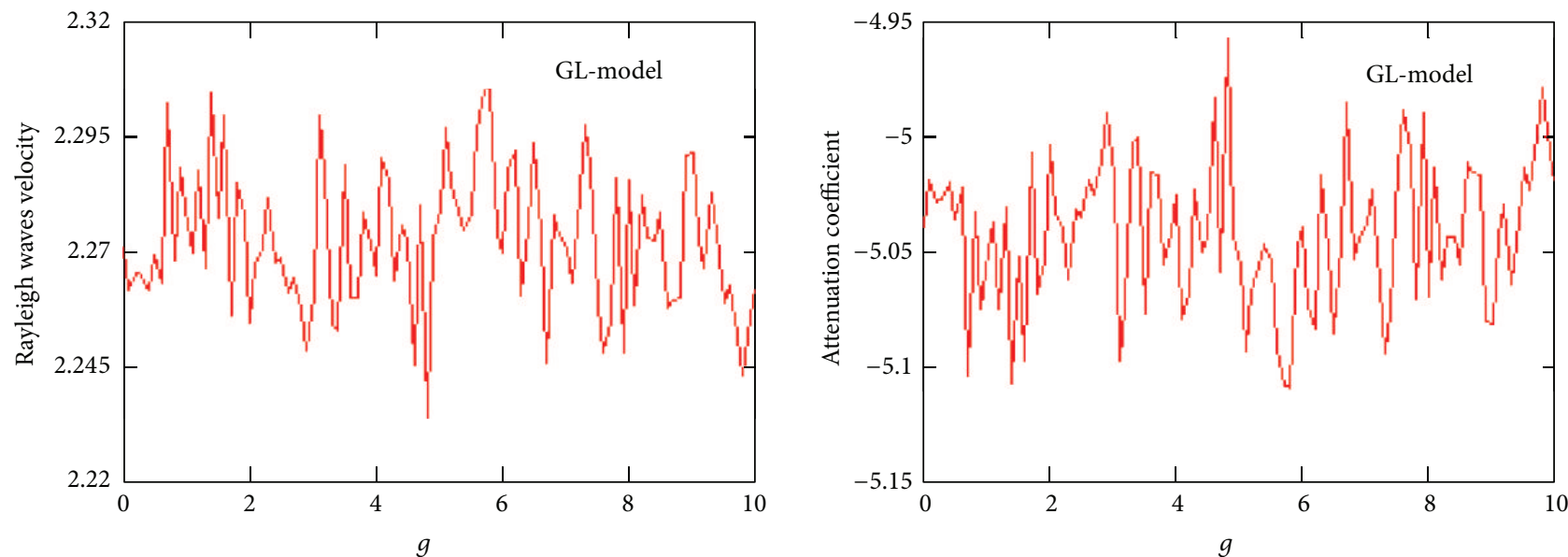

FIGURE 16: Variation of the Rayleigh waves velocity and attenuation coefficient (GL) model with respect to the gravity. 

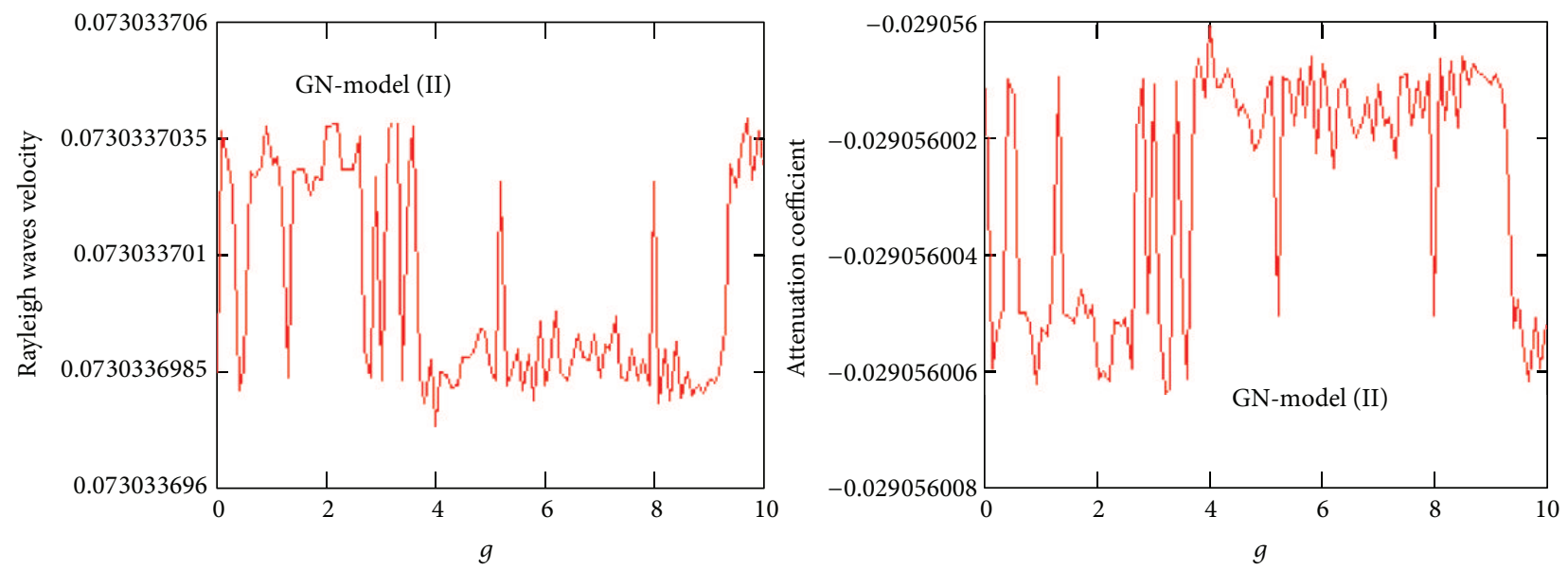

FIGURE 17: Variation of the Rayleigh waves velocity and attenuation coefficient (GN type (II)) model with respect to the gravity.
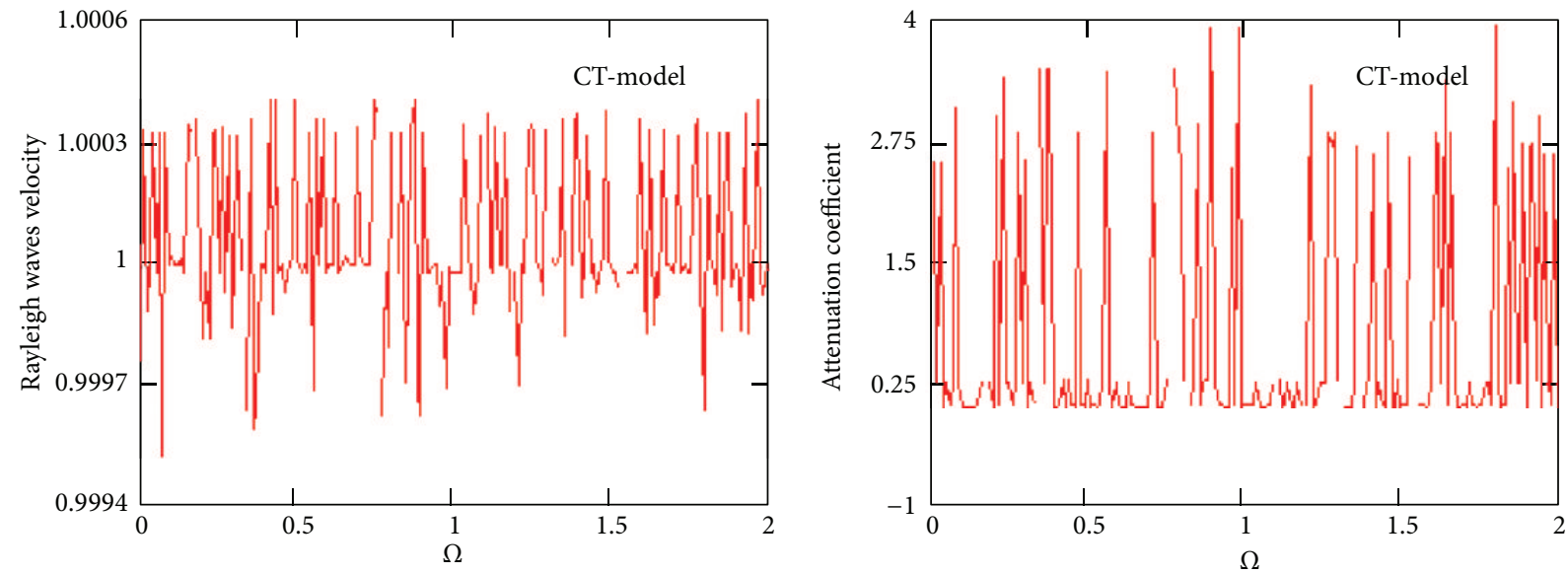

FIGURE 18: Variation of the Rayleigh waves velocity and attenuation coefficient CT-model with respect to the rotation.
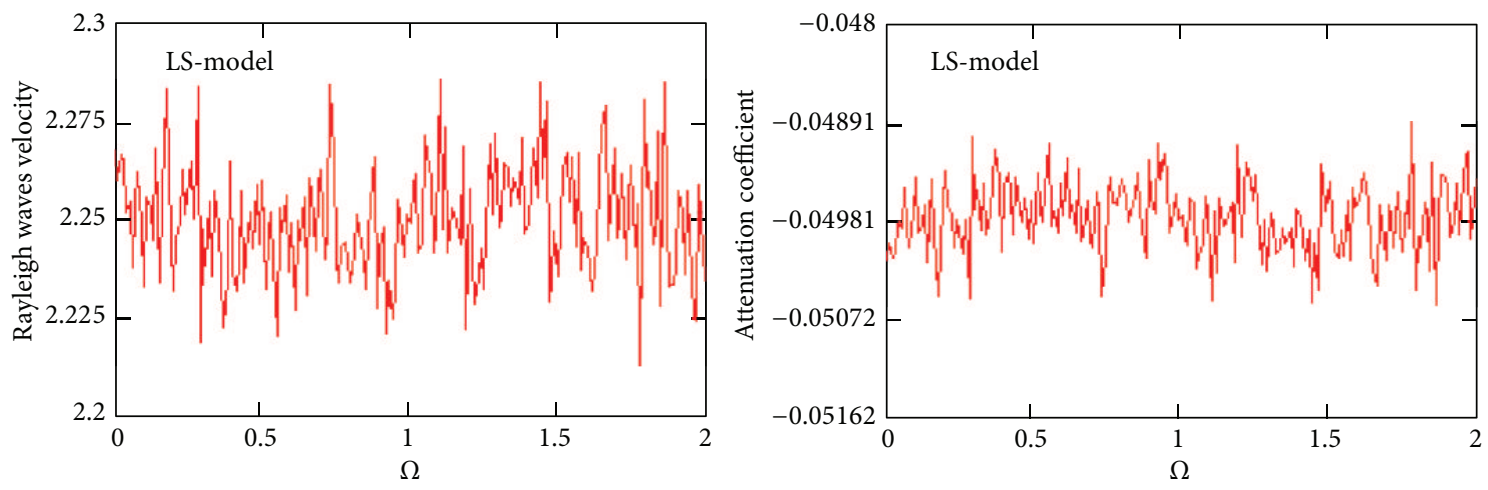

FIGURE 19: Variation of the Rayleigh waves velocity and attenuation coefficient LS-model with respect to the rotation.

Figure 9 shows the variation of the Stoneley secular equation with respect to phase velocity $c$, that has an oscillatory behavior of C-T theory, L-S theory, and G-L theory for different values of relaxation time $\tau_{0}$ in the whole range of the phase velocity $c$, while in G-N theory it decreases with increasing the constant $n^{*}$ and it increases with increasing phase velocity $c$. This is due to the fact that the thermal waves in the coupled theory travel with an infinite speed of propagation as opposed to a finite speed in the generalized case.

Figure 10 displays the variation of the Stoneley wave velocity and attenuation coefficient with respect to phase velocity $c$ that has oscillatory behavior in the whole range of $c$ for L-S theory, which changes from the positive to the negative gradually. 

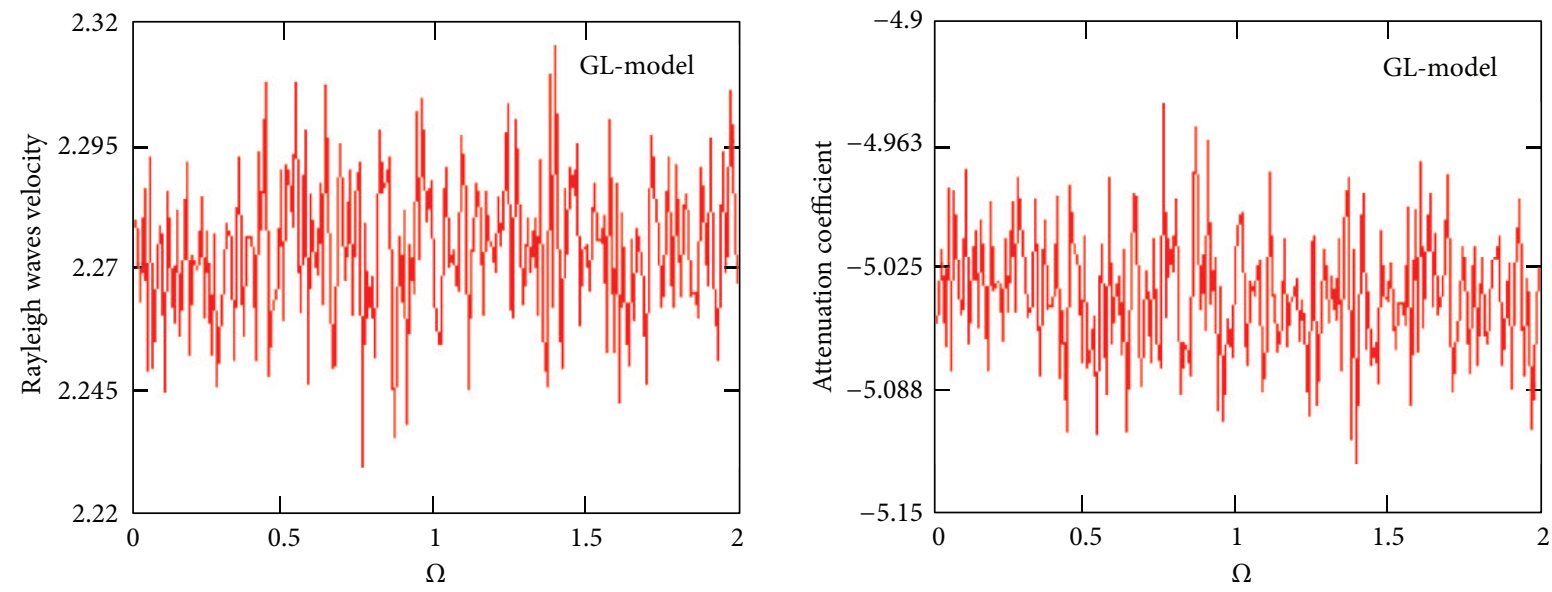

FIGURE 20: Variation of the Rayleigh waves velocity and attenuation coefficient GL-model with respect to the rotation.
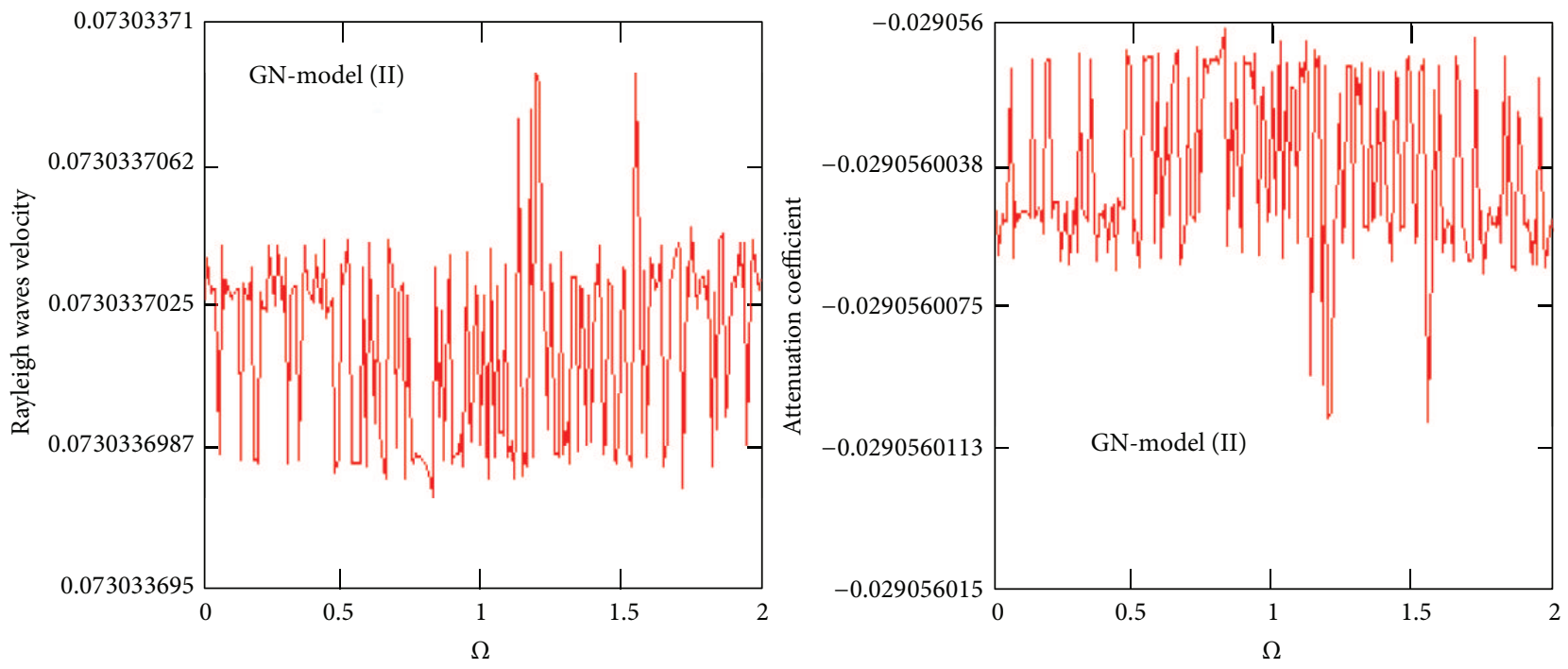

FIGURE 21: Variation of the Rayleigh waves velocity and attenuation coefficient (GN type (II)) model with respect to the rotation.

Figure 11 shows the variations of the Stoneley waves velocity and attenuation coefficient with respect to phase velocity $c$ that has oscillatory behavior in the whole range of $c$ for (G-L theory) that increases with increasing relaxation time $\tau_{0}, \theta_{0}$.

Figure 12 shows the variations of the Stoneley waves velocity and attenuation coefficient with respect to phase velocity $c$ for G-N theory in the whole range of $c$ for different values of the constant $n^{*}$ which changes from the positive to the negative gradually. In both figures, it is clear that the Stoneley wave velocity and attenuation coefficient have a nonzero value only in a bounded region of space. It is observed that the Stoneley wave velocity decreases with increasing the phase velocity, while the attenuation coefficient increases with increasing the constant $n^{*}$.

Figure 13 shows the variations of the Stoneley waves velocity and attenuation coefficient with respect to phase velocity $c$ for G-N theory in the whole range of $c$ for different values of constant $n^{*}$. In both figureures, it is clear that the Stoneley wave velocity and attenuation coefficient have a nonzero value only in a bounded region of space. It is observed that the Stoneley wave velocity increases with increasing the phase velocity, while it decreases with increasing constant $n^{*}$ and attenuation coefficient increases with increasing the phase velocity, while it increases with increasing constant $n^{*}$.

Figures $14,15,16,17,18,19,20$, and 21 show the variations of the Rayleigh velocity and attenuation coefficient with respect to gravity field $g$ and rotation $\Omega$, respectively, which has an oscillatory behavior in the whole range of $g$ and $\Omega$ for C-T theory, L-S theory, G-L theory, and G-N theory. These figureures indicate that the medium of attenuation coefficient along $g$ undergoes compressive deformation due to the thermal shock, except (C-T theory) it is a tension deformation. In both figureures, it is clear that the Rayleigh wave velocity and attenuation coefficient have a nonzero value only in a bounded region of space. Figure 22 shows the 


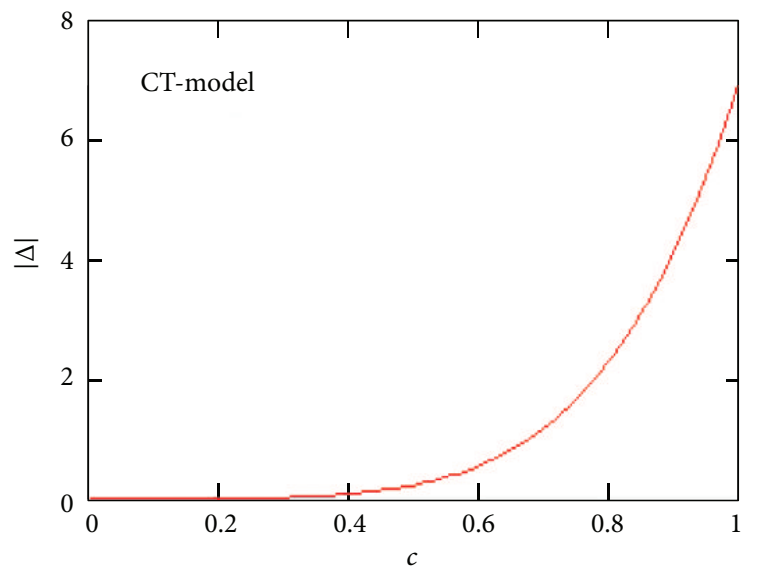

(a)

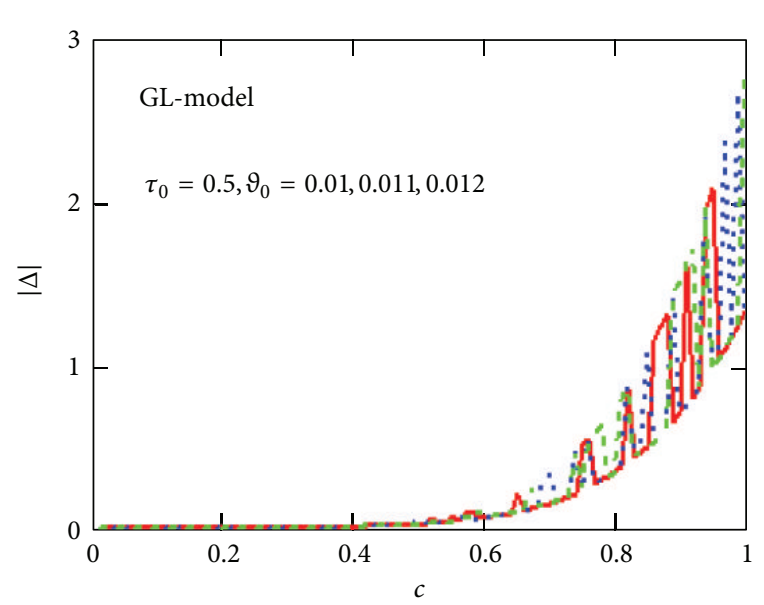

(c)

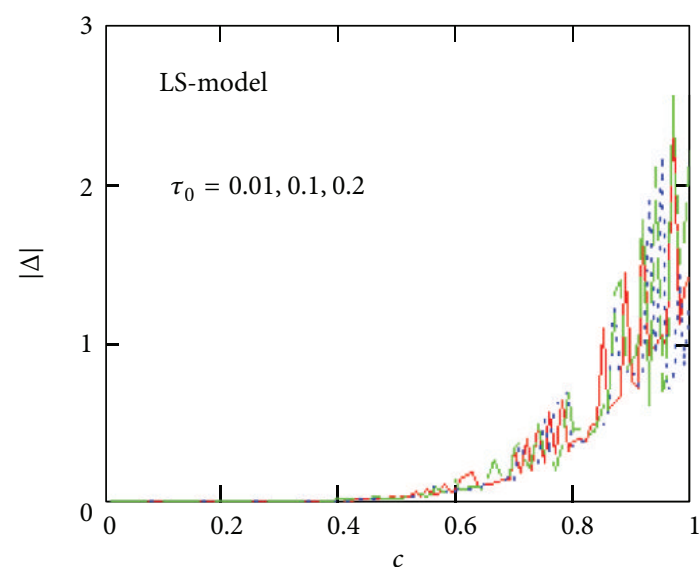

(b)

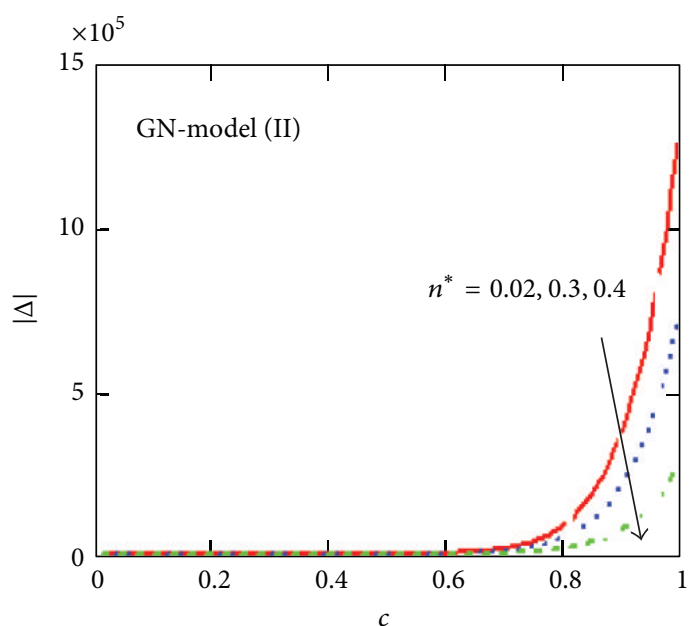

(d)

Figure 22: Variation of the Rayleigh secular equation for the (CT, LS, GL, and GN (type (II)) models with respect to the phase velocity.
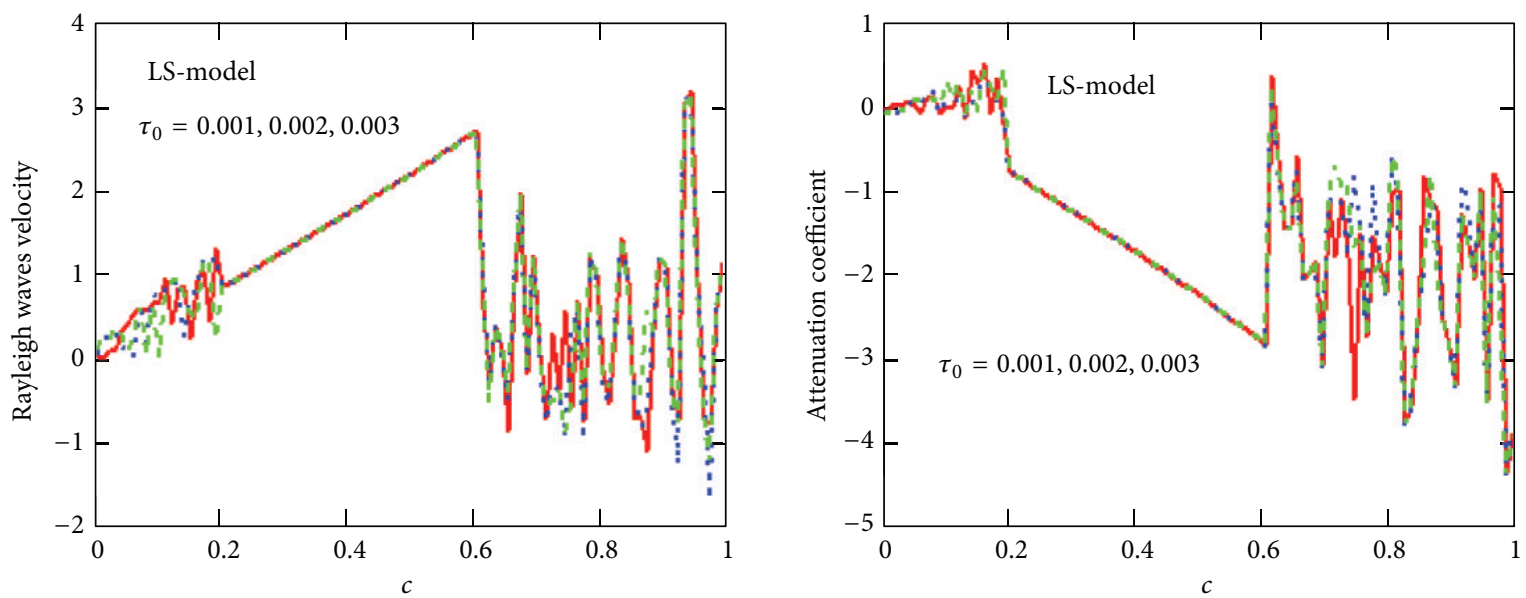

FIGURE 23: Variation of the Rayleigh waves velocity and attenuation coefficient (LS) model with respect to the phase velocity. 

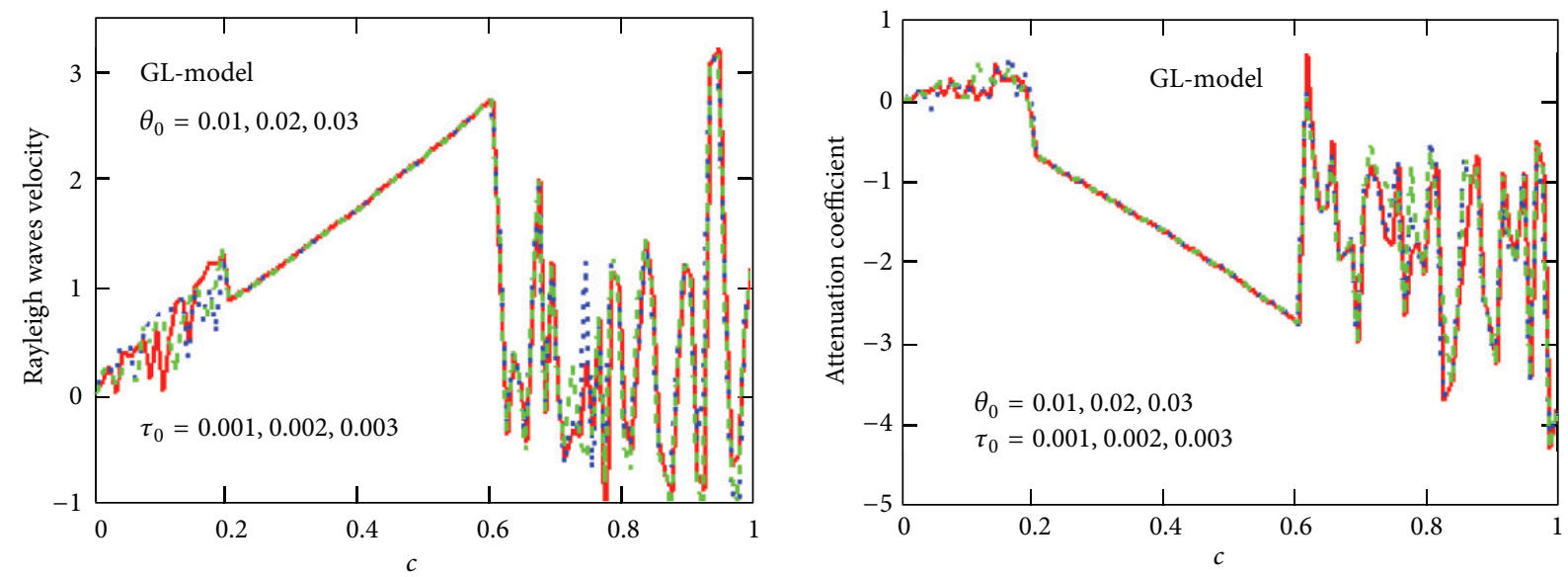

FIGURE 24: Variation of the Rayleigh waves velocity and attenuation coefficient (GL) model with respect to the phase velocity.
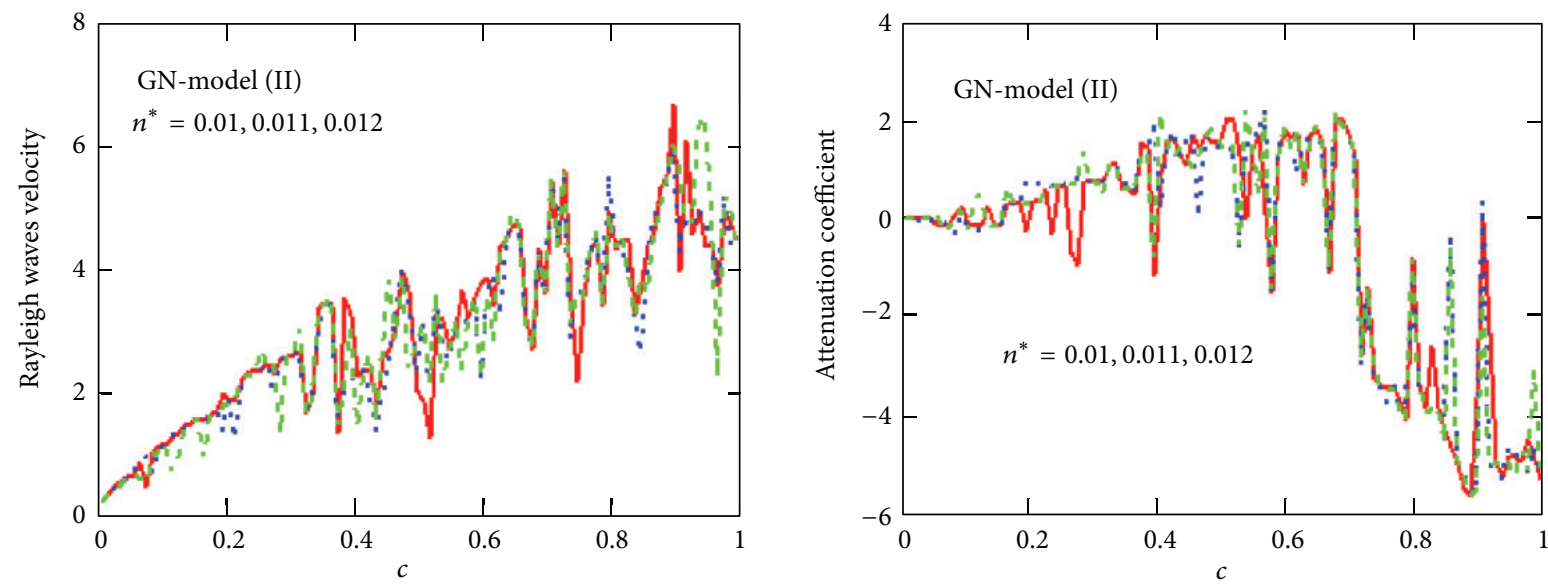

FIGURE 25: Variation of the Rayleigh waves velocity and attenuation coefficient (GN type (II)) model with respect to the phase velocity.
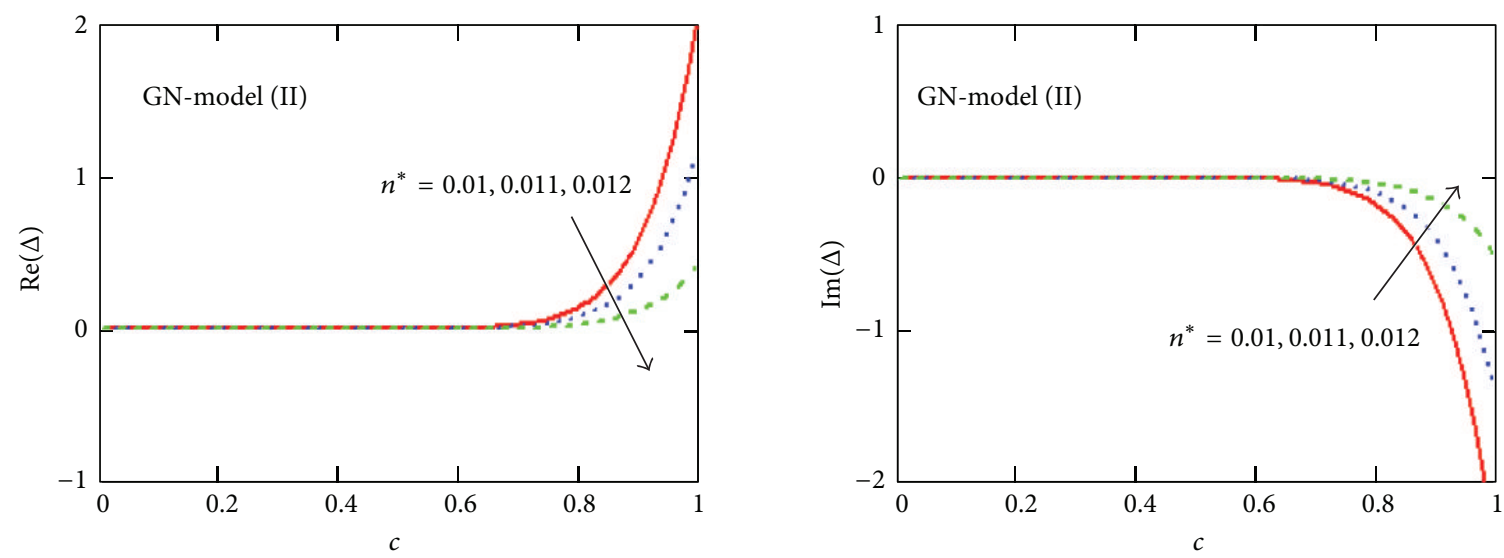

FIGURE 26: Variation of the Rayleigh waves velocity and attenuation coefficient (GN type (II)) model with respect to the phase velocity.

variations of the Rayleigh wave secular with respect to phase velocity $c$, it is increases with increasing the phase velocity for C-T theory, L-S theory, G-L theory, and G-N theory, while it decreases with increasing constant $n^{*}$ for G-N theory. In both figureures, it is clear that the Rayleigh waves secular equation has a nonzero value only in a bounded region of space.
Figures 23, 24, and 25 show the variations of the Rayleigh wave velocity and attenuation coefficient with respect to phase velocity $c$, it has oscillatory behavior in the whole range of $c$ for different values of relaxation times $\tau_{0}, \theta_{0}$, and $n^{*}$, respectively, for L-S theory, G-L theory, and G-N theory, which changes from the positive to the negative gradually. 


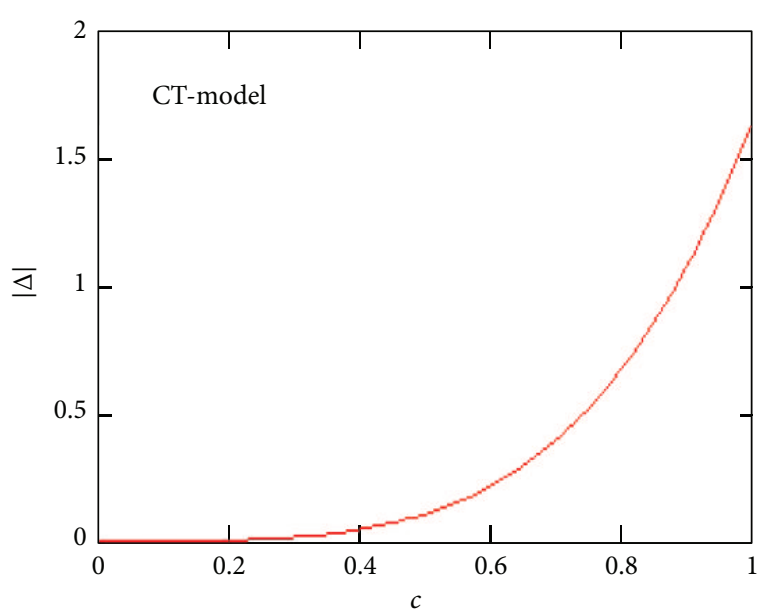

(a)

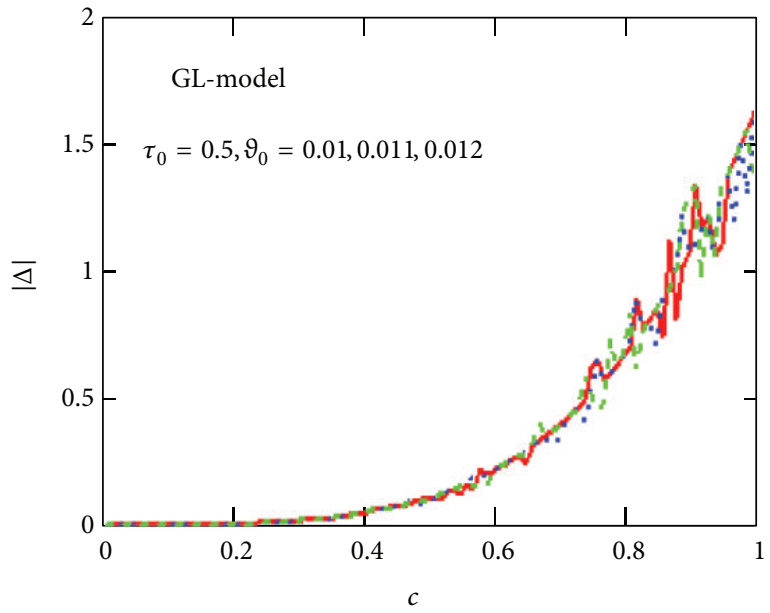

(c)

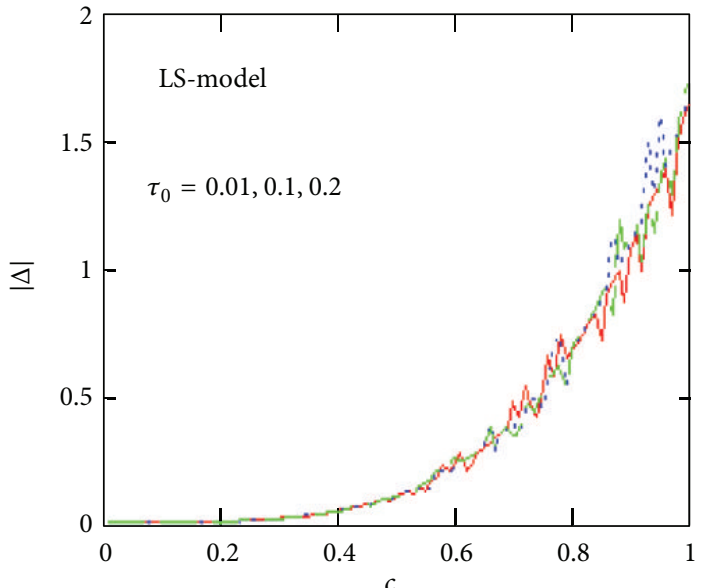

(b)

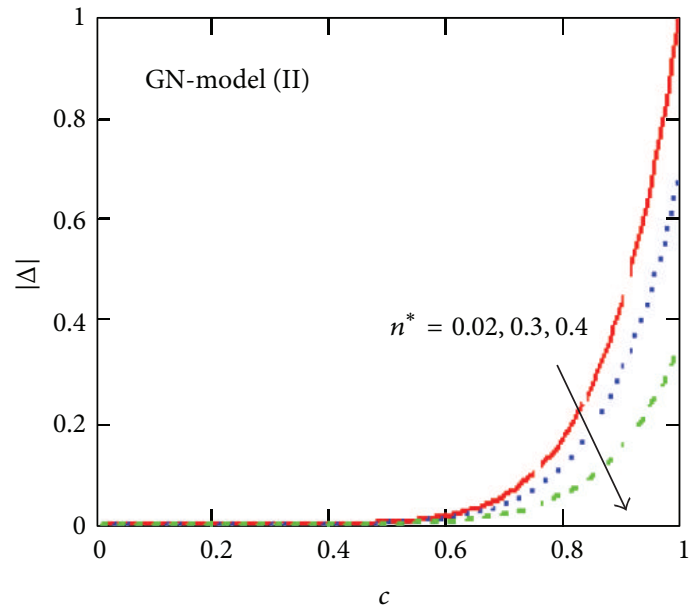

(d)

FIGURE 27: Variation of the Love secular equation for the (CT, LS, GL, and GN (type (II)) models with respect to the phase velocity.
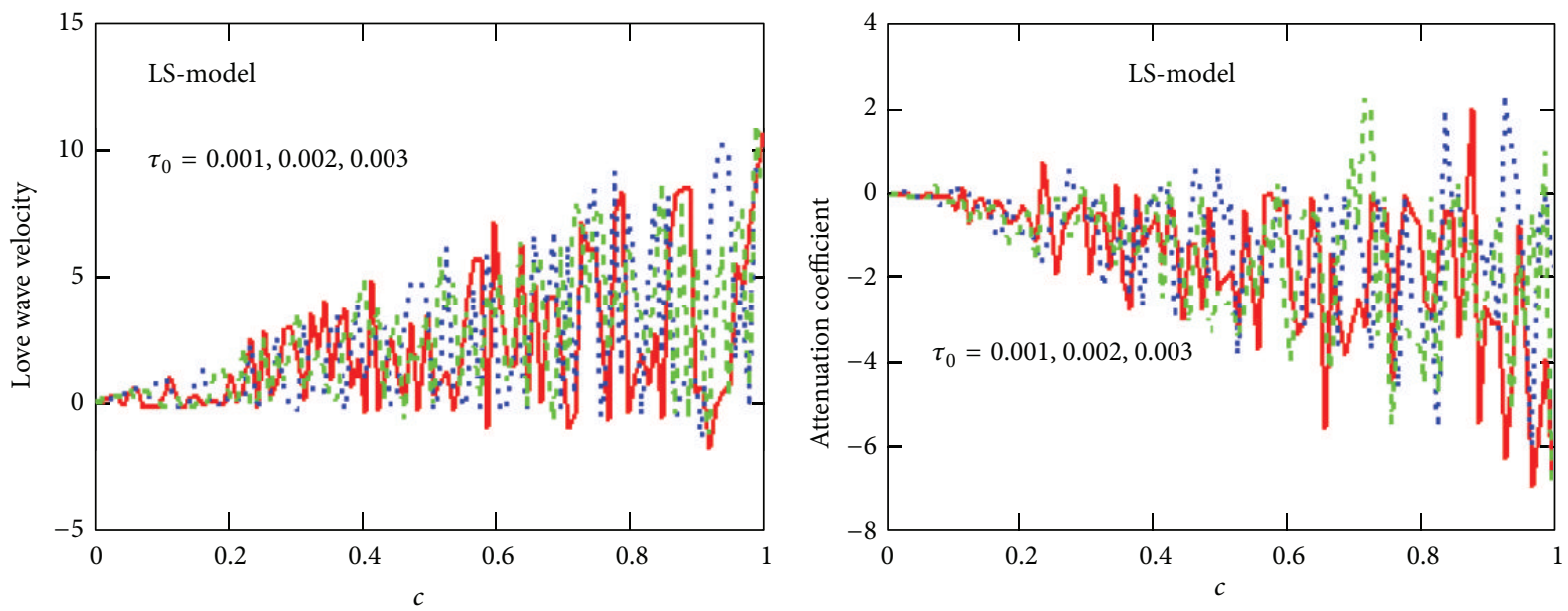

FIGURE 28: Variation of the Love waves velocity and attenuation coefficient (LS) model with respect to the phase velocity. 

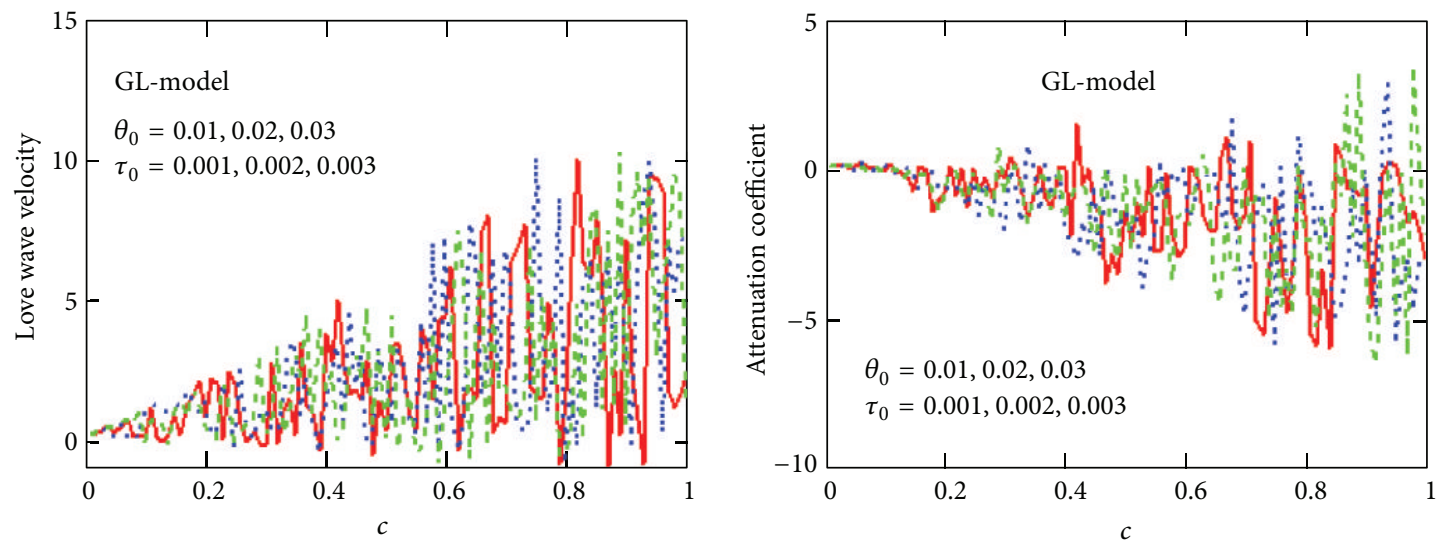

Figure 29: Variation of the Love waves velocity and attenuation coefficient (GL) model with respect to the phase velocity.
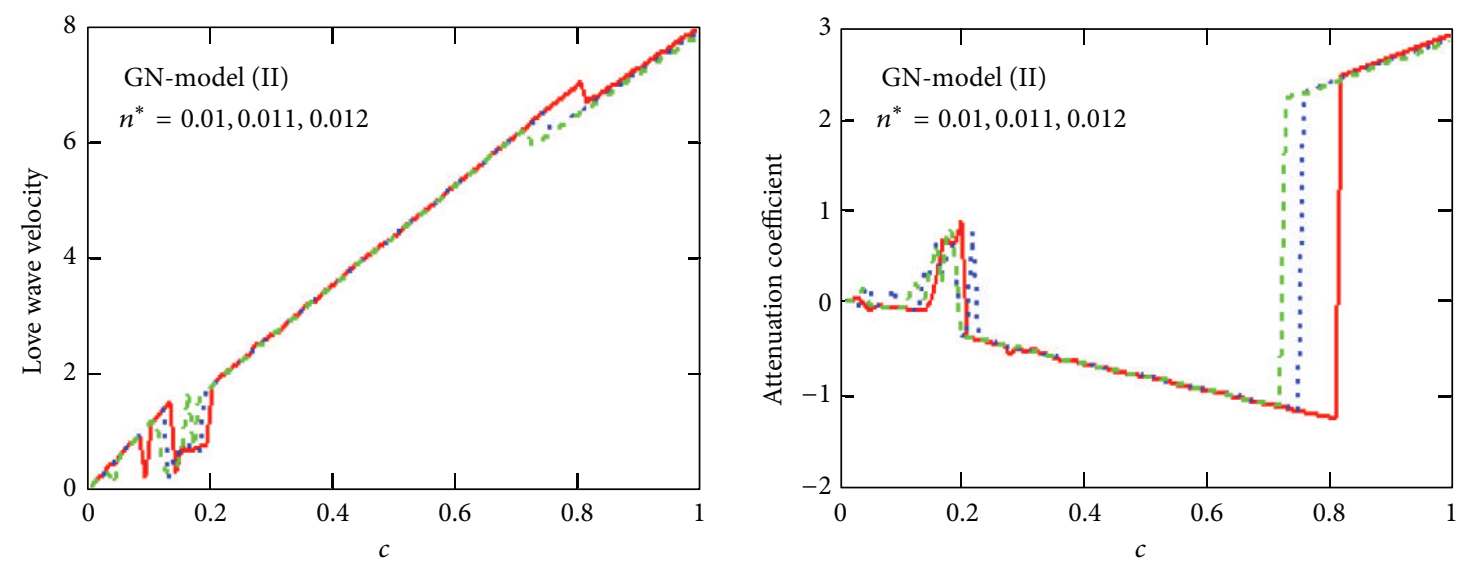

FIGURE 30: Variation of the Love waves velocity and attenuation coefficient (GN type (II)) model with respect to the phase velocity.

In both figureures, it is clear that the Rayleigh wave velocity and attenuation coefficient have a nonzero value only in a bounded region of space.

Figure 26 shows the variations of the Rayleigh wave velocity and attenuation coefficient with respect to phase velocity $c$ for G-N theory, the Rayleigh wave velocity increases with increasing phase velocity, while it decreases with increasing the constant $n^{*}$, and the attenuation coefficient decreases with increasing phase velocity and the constant $n^{*}$; it is clear that the Rayleigh wave velocity and attenuation coefficient have a nonzero value only in a bounded region of space.

Figure 27 shows the variation of the Love waves secular equation with respect to phase velocity $c$, which increases with increasing phase velocity for C-T theory, L-S theory, G$\mathrm{L}$ theory, and $\mathrm{G}-\mathrm{N}$ theory, while it decreases with increasing the constant $n^{*}$; it is clear that Love waves secular equation has a nonzero value only in a bounded region of space.

Figures 28, 29, and 30 show the variations of the Love wave velocity and attenuation coefficient with respect to phase velocity $c$, it has oscillatory behavior in the whole range of $c$ for different values of relaxation times $\tau_{0}, \theta_{0}$, and $n^{*}$, respectively, for L-S theory, G-L theory, and G-N theory, which changes from the positive to the negative gradually. In both figures, it is clear that Love wave velocity and attenuation coefficient have a nonzero value only in a bounded region of space.

\section{Conclusion}

Due to the complicated nature of the governing equations of the generalized thermoelasticity Fibre-reinforced theory, the work done in this field is unfortunately limited in number. The method used in this study provides a quite succesful in dealing with such problems. This method gives exact solutions in the generalized thermoelastic medium without any assumed restrictions on the actual physical quantities that appear in the governing equations of the problem considered. Important phenomena are observed in all these computations.

(i) It was found that for large values of time they give close results. The solutions obtained in the context of generalized thermoelasticity theory, however, exhibit the behavior of speed of surface wave propagation.

(ii) By comparing Figures 1-30, it was found that the surface wave velocity has the same behavior in both media. But with the passage of rotation, relaxation times, and gravity, numerical values of surface wave 
velocity in the thermoelastic medium are large in comparison due to the influences of gravity and relaxation times in the elastic medium.

(iii) Special cases are considered as Rayleigh waves, Love wave, and Stoneley surface waves in anisotropic generalized thermoelastic medium, as well as in the isotropic case.

(iv) The results presented in this paper should prove useful for researchers in material science and designers of new materials.

(v) Study of the phenomenon of rotation and gravity is also used to improve the conditions of oil extractions.

\section{References}

[1] A. M. Abd-Alla, S. M. Abo-Dahab, and H. A. H. Hammad, "Propagation of Rayleigh waves in generalized magneto-thermoelastic orthotropic material under initial stress and gravity field," Applied Mathematical Modelling, vol. 35, no. 6, pp. 29813000, 2011.

[2] A. M. Abd-Alla, G. A. Yahya, and A. M. Farhan, "Thermal stresses in an infinite circular cylinder," Journal of Mechanical Science and Technology, vol. 26, pp. 1829-1839, 2012.

[3] A. M. Abd-Alla and S. R. Mahmoud, "Magneto-thermoelastic problem in rotating non-homogeneous orthotropic hollow cylinder under the hyperbolic heat conduction model," Meccanica, vol. 45, no. 4, pp. 451-462, 2010.

[4] A. M. Abd-Alla and S. M. Ahmed, "Rayleigh waves in an orthotropic thermoelastic medium under gravity field and initial stress," Earth, Moon and Planets, vol. 75, no. 3, pp. 185-197, 1997.

[5] A. M. Abd-Alla, H. A. H. Hammad, and S. M. Abo-Dahab, "Rayleigh waves in a magnetoelastic half-space of orthotropic material under influence of initial stress and gravity field," Applied Mathematics and Computation, vol. 154, no. 2, pp. 583597, 2004.

[6] A. M. Elnaggar and A. M. Abd-Alla, "Rayleigh waves in magneto-thermo-microelastic half-space under initial stress," Earth, Moon and Planets, vol. 45, no. 2, pp. 175-185, 1989.

[7] A. M. Abd-Alla and S. M. Ahmed, "Rayleigh waves in an orthotropic thermoelastic medium under gravity field and initial stress," Earth, Moon and Planets, vol. 75, no. 3, pp. 185-197, 1997.

[8] A. M. Abd-Alla and S. M. Abo-Dahab, "Effect of rotation and initial stress on an infinite generalized magneto-thermoelastic diffusion body with a spherical cavity," Journal of Thermal Stresses, vol. 35, pp. 892-912, 2012.

[9] T.-T. Wu and J.-F. Chai, "Propagation of surface waves in anisotropic solids: theoretical calculation and experiment," Ultrasonics, vol. 32, no. 1, pp. 21-29, 1994.

[10] T.-T. Wu and Y.-H. Liu, "On the measurement of anisotropic elastic constants of fiber-reinforced composite plate using ultrasonic bulk wave and laser generated Lamb wave," Ultrasonics, vol. 37, no. 6, pp. 405-412, 1999.

[11] S.-H. Rhee, J.-K. Lee, and J.-J. Lee, “The group velocity variation of Lamb wave in fiber reinforced composite plate," Ultrasonics, vol. 47, no. 1-4, pp. 55-63, 2007.

[12] Y. B. Fu and Y. T. Zhang, "Continuum-mechanical modelling of kink-band formation in fibre-reinforced composites," International Journal of Solids and Structures, vol. 43, no. 11-12, pp. 3306-3323, 2006.
[13] H. D. Espinosa, S. Dwivedi, and H.-C. Lu, "Modeling impact induced delamination of woven fiber reinforced composites with contact/cohesive laws," Computer Methods in Applied Mechanics and Engineering, vol. 183, no. 3-4, pp. 259-290, 2000.

[14] Y. Weitsman and Y. Benveniste, "On wave propagation in materials reinforced with bi-directional fibers," Journal of Sound and Vibration, vol. 34, no. 2, pp. 179-198, 1974.

[15] Y. Weitsman, "On wave propagation and energy scattering in materials reinforced by inextensible fibers," International Journal of Solids and Structures, vol. 8, no. 5, pp. 627-650, 1972.

[16] H. L. Dai and X. Wang, "Stress wave propagation in piezoelectric fiber reinforced laminated composites subjected to thermal shock," Composite Structures, vol. 74, no. 1, pp. 51-62, 2006.

[17] T. Ohyoshi, "The propagation of Rayleigh waves along an obliquely cut surface in a directional fiber-reinforced composite," Composites Science and Technology, vol. 60, no. 12-13, pp. 2191-2196, 2000.

[18] G. A. Rogerson, "Penetration of impact waves in a six-ply fibre composite laminate," Journal of Sound and Vibration, vol. 158, no. 1, pp. 105-120, 1992.

[19] Y. Weitsrian, "On the reflection of harmonic waves in fiberreinforced materials," Journal of Sound and Vibration, vol. 26, no. 1, pp. 73-89, 1973.

[20] W. Huang, S. I. Rokhlin, and Y. J. Wang, "Effect of fibre-matrix interphase on wave propagation along, and scattering from, multilayered fibres in composites. Transfer matrix approach," Ultrasonics, vol. 33, no. 5, pp. 365-375, 1995.

[21] Y. B. Fu and Y. T. Zhang, "Continuum-mechanical modelling of kink-band formation in fibre-reinforced composites," International Journal of Solids and Structures, vol. 43, no. 11-12, pp. 3306-3323, 2006.

[22] B. Singh and S. J. Singh, "Reflection of plane waves at the free surface of a fibre-reinforced elastic half space," Sãdhanã, vol. 29, pp. 249-257, 2004.

[23] P. R. Sengupta and S. Nath, "Surface waves in fibre-reinforced anisotropic elastic media," Sãdhanã, vol. 26, pp. 363-370, 2001.

[24] S. K. Samal and R. Chattaraj, "Surface wave propagation in fiberreinforced anisotropic elastic layer between liquid saturated porous half space and uniform liquid layer," Acta Geophysica, vol. 59, no. 3, pp. 470-482, 2011.

[25] A. Chatopadhyay, R. L. K. Venkateswarlu, and SahaS, "Reflection of quasi-P and quasi-SV waves at the free and rigid boundaries of a fibre-reinforced medium," Sãdhanã, vol. 27, pp. 613-630, 2002.

[26] B. Singh, "Wave propagation in an incompressible transversely isotropic fibre-reinforced elastic media," Archive of Applied Mechanics, vol. 77, no. 4, pp. 253-258, 2007.

[27] B. Singh, "Wave propagation in thermally conducting linear fibre-reinforced composite materials," Archive of Applied Mechanics, vol. 75, no. 8-9, pp. 513-520, 2006.

[28] A. M. Abd-Alla, A. N. Abd-Alla, and N. A. Zeidan, "Thermal stresses in a nonhomogeneous orthotropic elastic multilayered cylinder," Journal of Thermal Stresses, vol. 23, no. 5, pp. 413-428, 2000.

[29] M. I. A. Othman and Kh. Lotfy, “The effect of magnetic field and rotation of the 2-D problem of a fiber-reinforced thermoelastic under three theories with influence of gravity," Mechanics of Material, vol. 60, pp. 129-143, 2013.

[30] A. E. Abouelregal and S. M. Abo-Dahab, "Dual phase lag model on magneto-thermoelasticity infinite non-homogeneous solid having a spherical cavity," Journal of Thermal Stresses, vol. 35, pp. 820-884, 2012. 
[31] A. M. Abd-alla and S. M. Abo-Dahab, "Effect of magnetic field on poroelastic bone model for internal remodeling," Applied Mathematics and Mechanics, vol. 34, pp. 889-906, 2013.

[32] A. M. Abd-Alla, S. M. Abo-Dahab, and F. S. Bayones, "Rayleigh waves in generalized magneto-thermo-viscoelastic granular medium under the influence of rotation, gravity field, and initial stress," Mathematical Problems in Engineering, vol. 2011, Article ID 763429, 47 pages, 2011.

[33] A. M. Abd-Alla, S. M. Abo-Dahab, and F. S. Bayones, "Propagation of Rayleigh waves in magneto-thermo-elastic half-space of a homogeneous orthotropic material under the effect of the rotation, initial stress and gravity field," Journal of Vibration and Control, vol. 19, no. 9, pp. 1395-1420, 2013.

[34] S. M. Abo-Dahab, "Propagation of Stoneley waves in magnetothermoelastic materials with voids and two relaxation times," Journal of Vibration and Control, 2014.

[35] P. M. Morse and H. Feshbach, Method of Theoretical Physics, Part I, McGraw-Hill, New York, NY, USA, 1953. 


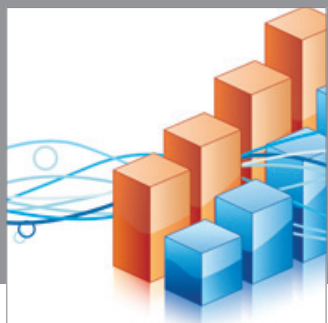

Advances in

Operations Research

mansans

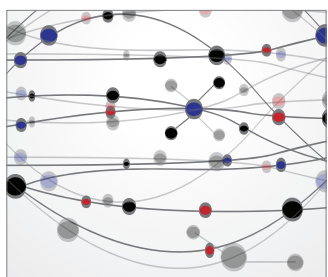

The Scientific World Journal
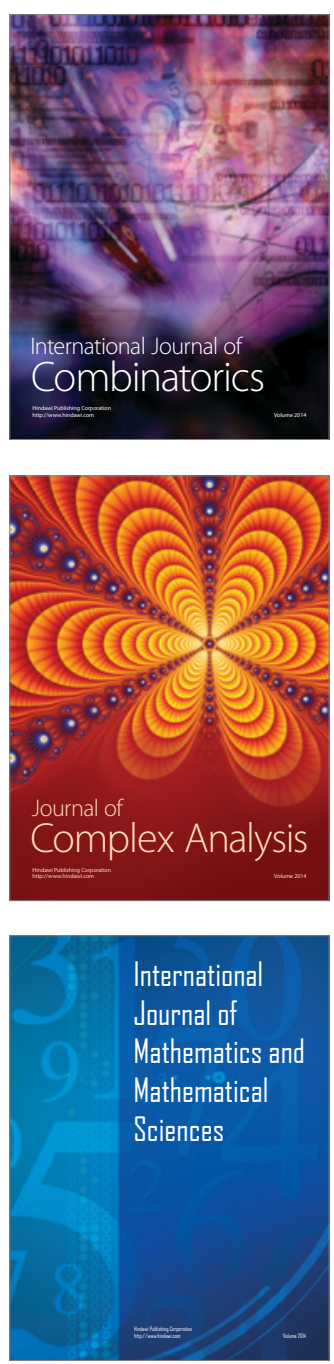
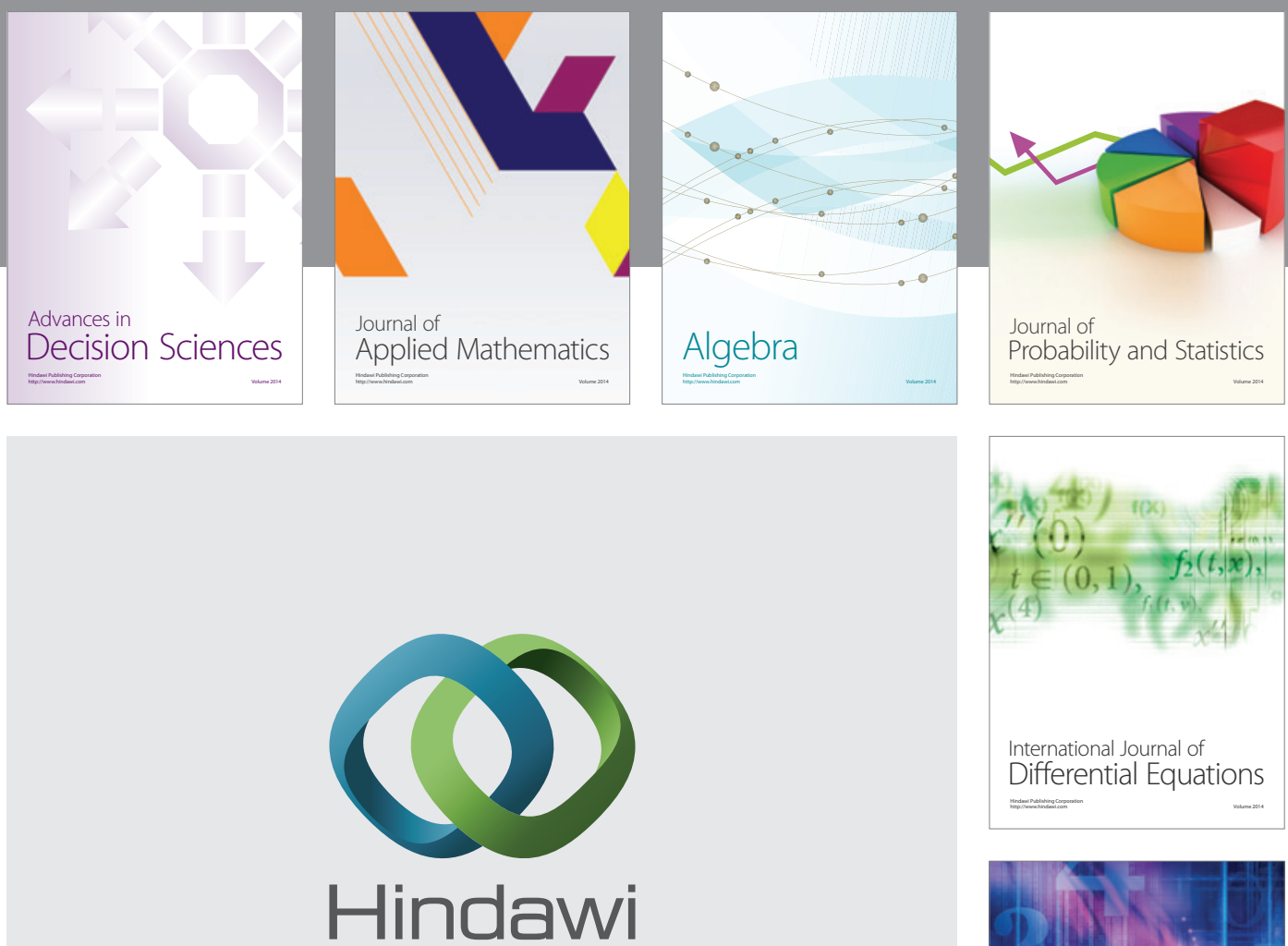

Submit your manuscripts at http://www.hindawi.com
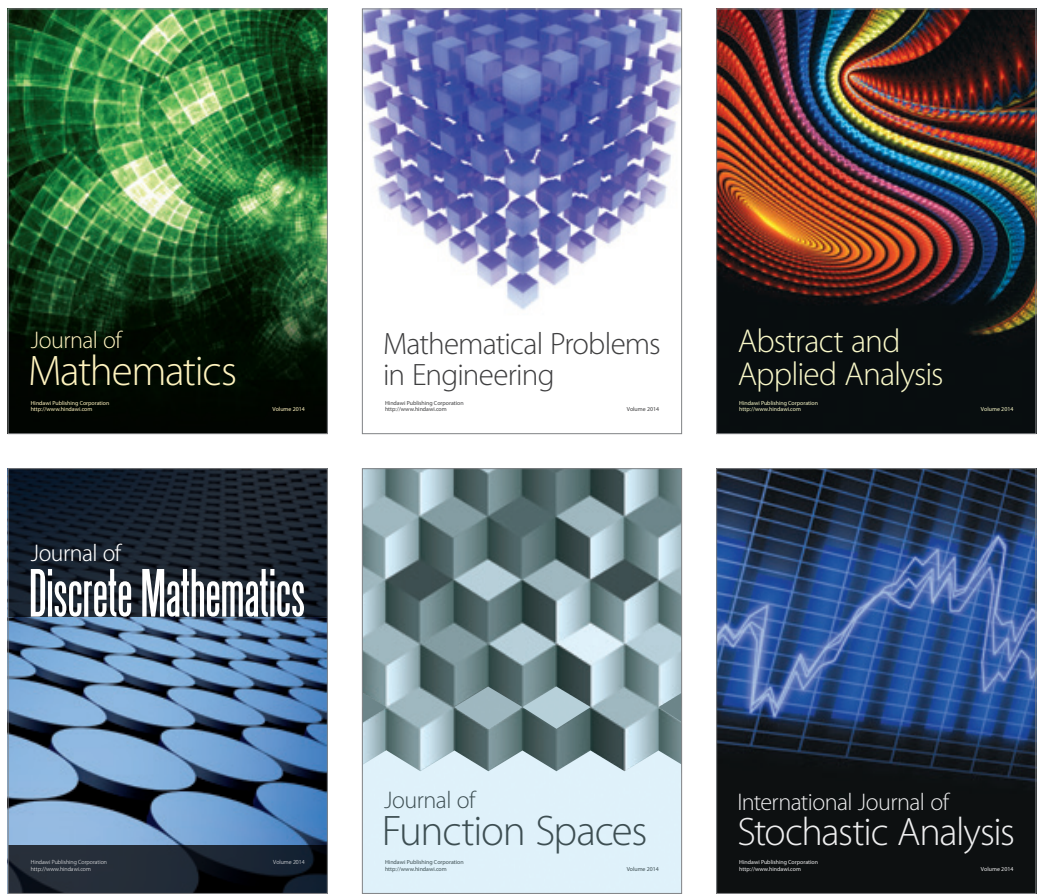

Journal of

Function Spaces

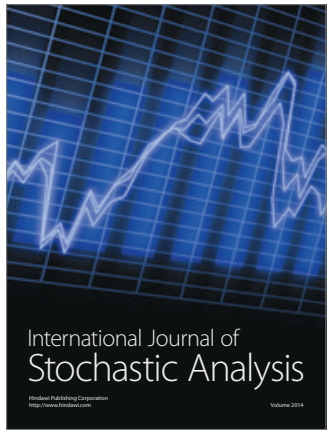

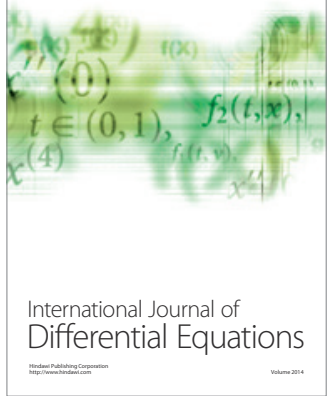
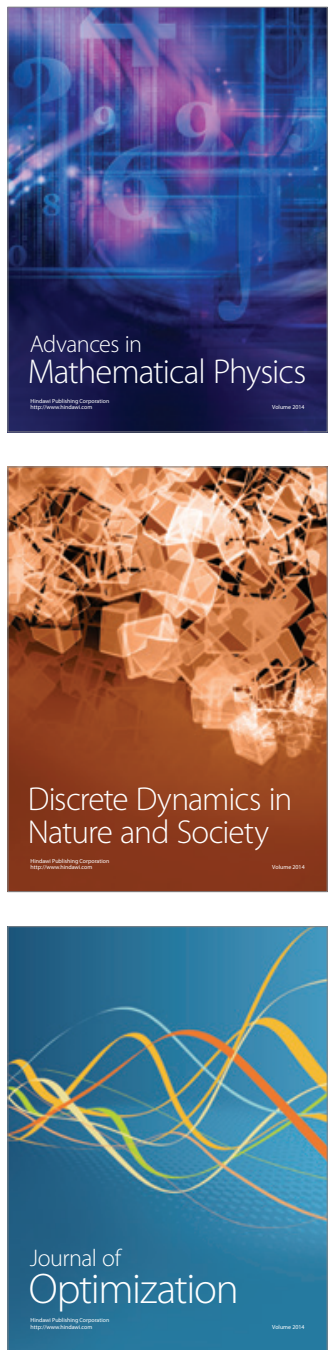